\title{
Searching for new theorems in M: a pure mathematical programming language
}

\author{
Open Mathematics Collaboration*广 \\ March 2, 2021
}

\begin{abstract}
We present a project to be deployed in Python to serve as a powerful auxiliar tool for theorem proving. In the future, it can be turned into a programming language in its own right.
\end{abstract}

keywords: m software, theorem prover, pure mathematics

The most updated version of this paper is available at https://osf.io/6gyq4/download

\section{Preamble}

1. This paper hasn't undergone peer review yet.

*All authors with their affiliations appear at the end of this paper.

†Corresponding author: mplobo@uft.edu.br|Open Mathematics Collaboration 


\section{Introduction}

2. A universal and unified scientific database, organized by precise and unique tags, searchable both by human and artificial intelligence, is possible, specially due to the advanced state of the art in the digital technologies we dispose at our fingertips nowadays [1-3].

3. Artificial intelligence, programmed by humans, might be able in the near future to inspect all axioms and theorems in pure mathematics so that new theorems can arise from it naturally.

\section{M language}

4. Hereafter, we refer to $\mathbf{M}$ as the programming language capable of analyzing, comparing, and drawing conclusions from theorems and axioms in pure mathematics.

\section{Stages of the project}

\section{A}

5. Deploy in python a search engine for pure mathematics.

6. In $\mathbf{M}$, theorems and axioms are searchable by tags.

\section{B}

7. Insert axioms and theorems, each with precise tags like in [4-8].

8. This stage consists of feeding a digital Open Mathematics Knowledge Base [4] into the $\mathbf{M}$ software.

9. (7) and (8) are performed by humans. 
10. Build a Super Graph (SG) [9-11] connecting all axioms that prove the theorems.

\section{$\mathrm{D}$}

11. Deploy an algorithm to find patterns in the SG, based on tags.

\section{$\mathrm{E}$}

12. This stage is devoted to artificial intelligence.

13. The program should read new theorems and axioms and add pieces of codes into itself.

14. Subsequently, it searches for new patterns and theorems using machine learning.

\section{Objects in $\mathrm{M}$}

15. The following commands take into account only the mathematical objects added into the knowledge base (check add.ipynb in the Appendix).

16. tags ( ) shows all searchable tags.

17. s('primes') accomplishes a simple search for the tag "primes" (it shows all mathematical objects regarding prime numbers ).

18. proof ('primes') shows mathematical proofs about prime numbers.

19. m('primes') searches for the tag "primes" (it shows all mathematical objects that need prerequisites). 


\section{In the Appendix}

20. All files are available for download here [14] (subfolder "195") or by the following links.

21. omkb.py

Python library (the search engine)

https://osf.io/9shbg

22. add.ipynb

Jupyter notebook that adds content into the mathematical knowledge base.

https://osf.io/b3f6h/

23. example.ipynb

A Jupyter notebook with some examples.

https://osf.io/qxpw5/

\section{Final Remarks}

24. We hope that the $\mathbf{M}$ language in the future ultimately be consisted of a huge mathematical database comprising axioms and theorems from nearly all areas in addition to a highly intelligent algorithm programmed for searching new connections in the realm of the so vast mathematical world.

25. If the mathematical community embraces this very audacious project, the chances of success are high.

\section{Open Invitation}

Review, add content, and co-author this paper $[12,13]$.

Join the Open Mathematics Collaboration.

Send your contribution to mplobo@uft.edu.br. 


\section{Open Science}

The latex file for this paper together with other supplementary files are available [14].

\section{Ethical conduct of research}

This original work was pre-registered under the OSF Preprints [15]; please cite it accordingly [16]. This will ensure that researches are conducted with integrity and intellectual honesty at all times and by all means.

\section{Acknowledgements}

+ Center for Open Science https://cos.io

+ Open Science Framework https://osf.io

\section{Agreement}

26. All authors agree with [13].

\section{References}

[1] Lobo, Matheus P. "Scientific Tags." OSF Preprints, 6 May 2020. https://doi.org/10.31219/osf .io/tahx5

[2] Lobo, Matheus P. "Universal Knowledge Base." OSF Preprints, 21 June 2020. https://doi .org/10.31219/osf .io/pwvzt 
[3] Lobo, Matheus P. "Open Knowledge Base from Resources and Units of Knowledge." OSF Preprints, 17 May 2020.

https://doi.org/10.31219/osf .io/7vayt

[4] Lobo, Matheus P. "Open Mathematics Knowledge Base." OSF Preprints, 13 May 2020.

https://doi.org/10.31219/osf .io/evq8a

[5] Lobo, Matheus P. "Mathematical Tags of Group Theory." OSF Preprints, 10 May 2020.

https://doi.org/10.31219/osf .io/9xk68

[6] Lobo, Matheus P. "A List of Notations and Mathematical Definitions for Abstract Algebra Organized by Tags." OSF Preprints, 6 May 2020. https://doi.org/10.31219/osf .io/u8yx2

[7] Calcina, Sabrina G. S. "Test Functions and Distributions (open Mathematics Knowledge Base)." OSF Preprints, 23 July 2020.

https://doi.org/10.31219/osf .io/xne52

[8] Hancco, Alvaro J. Y. "Differential Forms (open Mathematics Knowledge Base)." OSF Preprints, 19 July 2020.

https://doi.org/10.31219/osf .io/g7uqb

[9] West, Douglas Brent. Introduction to graph theory. Vol. 2. Upper Saddle River, NJ: Prentice hall, 1996.

[10] Bollobás, Béla. Modern graph theory. Vol. 184. Springer Science \& Business Media, 2013.

[11] Biggs, Norman, E. Keith Lloyd, and Robin J. Wilson. Graph Theory, 1736-1936. Oxford University Press, 1986.

[12] Lobo, Matheus P. "Microarticles." OSF Preprints, 28 Oct. 2019. https://doi.org/10.31219/osf.io/ejrct 
[13] Lobo, Matheus P. "Simple Guidelines for Authors: Open Journal of Mathematics and Physics." OSF Preprints, 15 Nov. 2019.

https://doi.org/10.31219/osf .io/fk836

[14] Lobo, Matheus P. "Open Journal of Mathematics and Physics (OJMP)." OSF, 21 Apr. 2020.

https://doi.org/10.17605/osf .io/6hzyp

https://osf.io/6hzyp/files

[15] COS. Open Science Framework. https://osf.io

[16] Lobo, Matheus P. "Searching for New Theorems in M: A Pure Mathematical Programming Language." OSF Preprints, 31 Dec. 2020. https://doi.org/10.31219/osf .io/6gyq4

\section{The Open Mathematics Collaboration}

Matheus Pereira Lobo (lead author, mplobo@uft.edu.br) $)^{1,2}$ https://orcid.org/0000-0003-4554-1372

Sergio Williams Ferreira de Sousa ${ }^{1}$

(programmer, wllyvn@gmail.com)

${ }^{1}$ Federal University of Tocantins (Brazil)

${ }^{2}$ Universidade Aberta (UAb, Portugal) 


\section{example}

January 17, 2021

\subsection{Example of $M$}

First, import the following library.

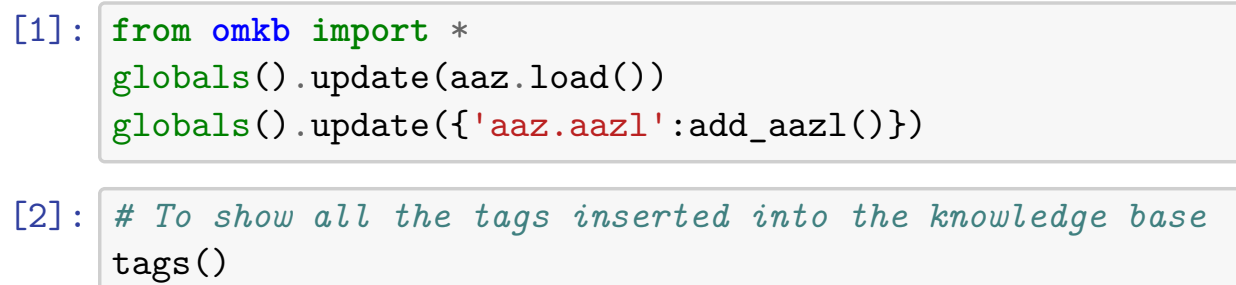

1. +

2. $:=$

3. :

4. L-símbolo

5. alcance

6. aridade

7. atômica

8. axiom

9. axioma

10. cadeia

11. caso base

12. caso indutivo

13. complexidade

14. conectivo

15. conjecture

16. conjunto

17. constante

18. definição

19. equation

20. equivalente

21. espaço

22. especificar

23. estrutura

24. estrutura matemática

25. even

26. exemplo
27. factorial

28. finite

29. função

30. função n-ária

31. fórmula

32. fórmula atômica

33. goldbach

34. grupo

35. identidade

36. igualdade

37. induction

38. indução

39. infinite

40. inversa

41. linguagem

42. mais

43. metalinguístico

44. modelo

45. n-upla

46. n-ária

47. no prime

48. notação

49. notação polonesa

50. número real

51. operação binária

52. parêntesis
53. primeira ordem

54. primes

55. primitiva

56. proof

57. prova

58. quantificador

59. real

60. recursão

61. relação

62. relação n-ária

63. sequence

64. sequência

65. simples

66. sintaxe

67. sistema

68. subfórmula

69. símbolo

70. símbolo constante

71. termo

72. theorem

73. trio ordenado

74. upla

75. variable

76. variável 

$\hookrightarrow$ objects $\operatorname{tags}(1)$

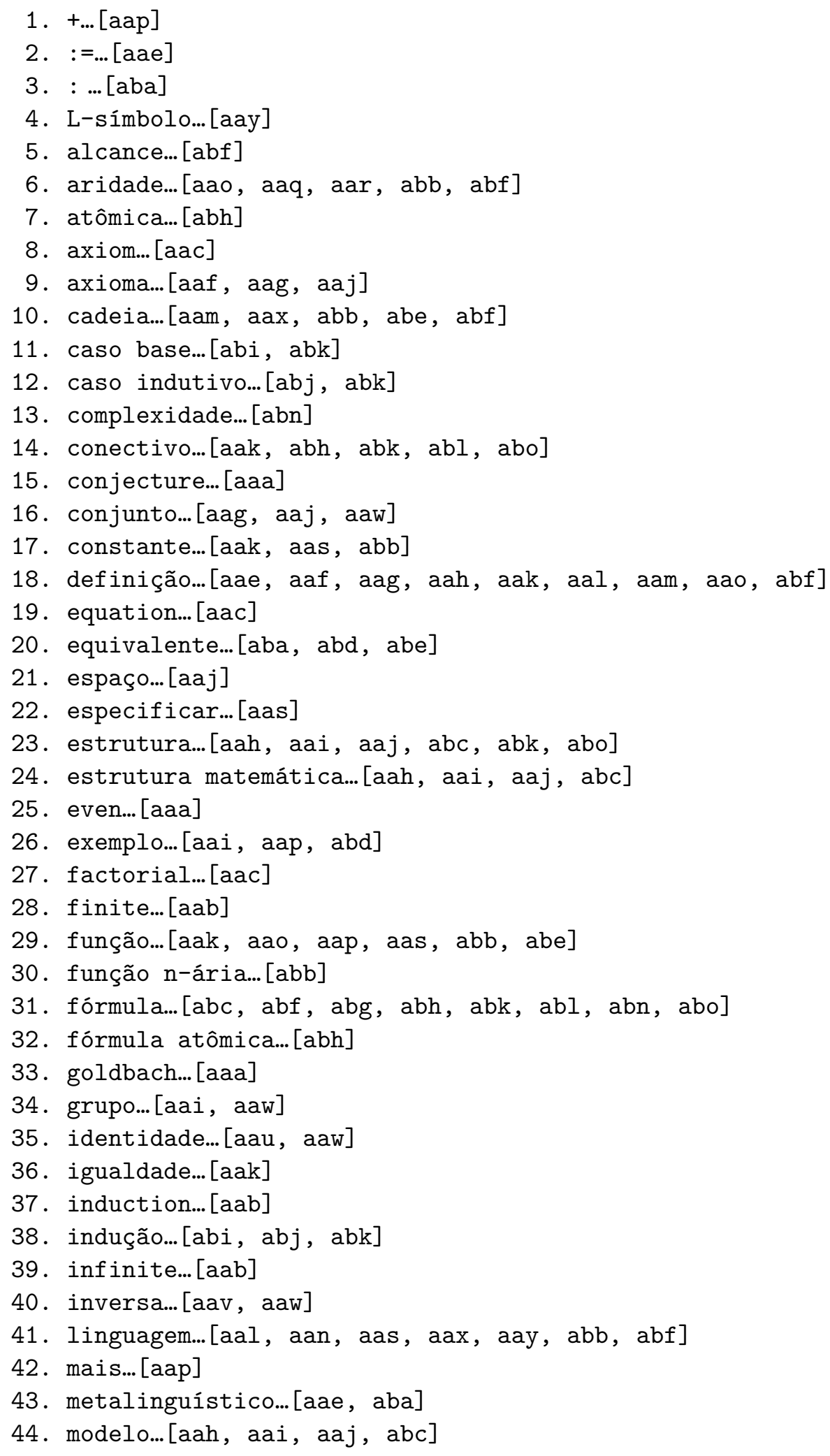


45. n-upla...[aaq, aar]

46. n-ária...[aao, aar, abb, abf]

47. no prime...[aad]

48. notação...[aae, aak, aba, abd, abe]

49. notação polonesa... [abd, abe, abf]

50. número real...[aaj]

51. operação binária...[aat, aaw]

52. parêntesis...[aak]

53. primeira ordem...[aan]

54. primes...[aaa, aad]

55. primitiva... [abh]

56. proof...[aab]

57. prova... [abi, abj]

58. quantificador...[aak, abh, abk, abl, abo]

59. real...[aaj]

60. recursão... [abg, abh]

61. relação...[aak, aar, aas, abf]

62. relação n-ária... [abf]

63. sequence... [aad]

64. sequência... [aam]

65. simples...[abh, abl]

66. sintaxe... [aal]

67. sistema... [aag]

68. subfórmula... [abl, abn]

69. símbolo...[aae, aak, aal, aam, aan, aap, aas, aax, aay, aba, $\mathrm{abb}, \mathrm{abe}, \mathrm{abf}]$

70. símbolo constante... [abb]

71. termo...[aax, abb, abf]

72. theorem... [aad]

73. trio ordenado...[aaj]

74. upla... [aaq, aar]

75. variable...[aac]

76. variável... [aak, aap, abb, abf]

[4]: s('goldbach')

tags: goldbach

results: 1 from 1

goldbach:

aaa [conjecture, goldbach, primes, even]

$\forall n \in \mathbb{N}_{>1}:(m=2 n) \rightarrow\left(m=p_{i}+p_{j}\right)$

[5]: s('primes/conjecture')

tags: primes, conjecture 
results: 2 from 2

conjecture, primes:

aaa [conjecture, goldbach, primes, even]

$\forall n \in \mathbb{N}_{>1}:(m=2 n) \rightarrow\left(m=p_{i}+p_{j}\right)$

primes:

aad [theorem, primes, sequence, no prime]

$\forall n \in \mathbb{N}_{>0}, \exists\left(a_{1}, a_{2}, \ldots, a_{n}\right)=$ sequence of natural numbers with no prime

[6]: s('primes/theorem')

tags: theorem, primes

results: 2 from 2

theorem, primes:

aad [theorem, primes, sequence, no prime]

$\forall n \in \mathbb{N}_{>0}, \exists\left(a_{1}, a_{2}, \ldots, a_{n}\right)=$ sequence of natural numbers with no prime

primes:

aaa [conjecture, goldbach, primes, even]

$\forall n \in \mathbb{N}_{>1}:(m=2 n) \rightarrow\left(m=p_{i}+p_{j}\right)$

[7] : proof ('primes')

for the tags: primes

results: 2 de 2

$\mathrm{aab}, \mathrm{aac} \sim>$ aad

aad: [theorem, primes, sequence, no prime]

$\forall n \in \mathbb{N}_{>0}, \exists\left(a_{1}, a_{2}, \ldots, a_{n}\right)=$ sequence of natural numbers with no prime

$a a b$ : [proof, induction, infinite, finite]

induction

aac: [axiom, variable, equation, factorial]

$x=(n+1) !+2$ 
$\sim$ aaa

aaa: [conjecture, goldbach, primes, even]

$\forall n \in \mathbb{N}_{>1}:(m=2 n) \rightarrow\left(m=p_{i}+p_{j}\right)$

[8]: m('linguagem')

for the tags: linguagem

aal: [sintaxe, linguagem]

sintaxe $:=$ símbolos (de uma linguagem)

aak:

\section{CATEGORIAS DE SÍMBOLOS}

Parêntesis: $($,

Conectivos: $\vee \neg \neg$

Quantificador: $\forall$

Variáveis (uma para cada inteiro positivo $n$ ): $v_{1}, v_{2}, \ldots, v_{n}, \ldots$

Vars $:=$ conjunto de símbolos de variáveis

Igualdade: $=$

Constante: para cada $n$ inteiro positivo, algum conjunto contendo zero ou mais símbolos

Função: para cada $n$ inteiro positivo, algum conjunto contendo zero ou mais símbolos de funções $n$-árias

Relação: para cada $n$ inteiro positivo, algum conjunto contendo zero ou mais símbolos de relações $n$-árias

aae :

O símbolo := indica que o que aparece à esquerda é definido como o que aparece à direita.

aan: [linguagem, primeira ordem]

linguagem de primeira ordem $:=$ coleção infinita de símbolos distintos (tal que nenhum esteja contido no outro)

aak:

CATEGORIAS DE SÍMBOLOS

Parêntesis: $($,

Conectivos: $\vee, \neg$ 
Quantificador: $\forall$

Variáveis (uma para cada inteiro positivo $n$ ): $v_{1}, v_{2}, \ldots, v_{n}, \ldots$

Vars $:=$ conjunto de símbolos de variáveis

Igualdade: $=$

Constante: para cada $n$ inteiro positivo, algum conjunto contendo zero ou mais símbolos

Função: para cada $n$ inteiro positivo, algum conjunto contendo zero ou mais símbolos de funções $n$-árias

Relação: para cada $n$ inteiro positivo, algum conjunto contendo zero ou mais símbolos de relações $n$-árias

aae:

O símbolo := indica que o que aparece à esquerda é definido como o que aparece à direita.

aas: [especificar, linguagem]

especificar uma linguagem := listar os símbolos (constante, função, relação)

[ ]: 


\section{add}

January 18, 2021

[12] :

from omkb import *

globals () . update (aaz . load())

globals() . update (\{'aaz.aazl' :add_aazl() $\})$

[13]: \# aaz

\# $a b c=a a z$ ('abc', latex, tags, tags_name, derivation, dependent, references)

$\# a a a=a a z\left({ }^{\prime} a a a^{\prime}, r^{\prime} ',[],[],[],[],[]\right)$

\subsection{AAA: Goldbach's Conjecture}

$\forall n \in \mathbb{N}_{>1}:(m=2 n) \rightarrow\left(m=p_{i}+p_{j}\right)$

[14]: aaa $=\operatorname{aaz}\left(\right.$ 'aaa' $^{\prime}, r^{\prime} \backslash$ forall $n \backslash$ in $\backslash$ mathbb $\mathbb{N}_{-}\{>1\}:(m=2 n) \backslash r i g h t a r r o w\left(m=p_{-} i+p_{-} j\right){ }^{\prime}$, ['conjecture', ' 'goldbach', 'primes', ' even'], ['conjecture' , 'goldbach'] ) aaa. save()

aaa ()

print (aaa.tags)

aaa.tags_name

saved

$\forall n \in \mathbb{N}_{>1}:(m=2 n) \rightarrow\left(m=p_{i}+p_{j}\right)$

['conjecture', 'goldbach', 'primes', 'even']

[14]: ['conjecture', 'goldbach']

\subsection{AAB: Proof by Induction}

induction

[15]: $\mathrm{aab}=\mathrm{aaz}\left({ }^{\prime} \mathrm{aab}{ }^{\prime}, \mathrm{r}^{\prime} \backslash \operatorname{mbox}\{\text { induction }\}\right\}^{\prime}$,

$\hookrightarrow[$ ['proof ', 'induction' , 'infinite' , 'finite'] , ['proof ' , 'induction'] , [abi, abj] , []) aab.save()

$\mathrm{aab}()$

print (aab.tags)

aab.tags_name

saved 
induction

['proof', 'induction', 'infinite', 'finite']

[15]: ['proof', 'induction']

\subsection{AAC: Factorial (equation)}

$x=(n+1) !+2$

[16]: aac $=\operatorname{aaz}\left({ }^{\prime} \operatorname{aac}^{\prime}, r^{\prime} x=(n+1) !+2 '\right.$, ['axiom', 'variable' , 'equation' , 'factorial'], ['equation' , 'factorial'])

aac.save()

aac ()

print (aac.tags)

aac.tags_name

saved

$x=(n+1) !+2$

['axiom', 'variable', 'equation', 'factorial']

[16]: ['equation', 'factorial']

\subsection{AAD: Sequence with no primes (theorem)}

$\forall n \in \mathbb{N}_{>0}, \exists\left(a_{1}, a_{2}, \ldots, a_{n}\right)=$ sequence of natural numbers with no prime

[74]: aad = aaz('aad', $r^{\prime} \backslash$ forall $n \backslash$ in $\backslash$ mathbb $N_{-}\{>0\}, \backslash$ exists $\left(a_{-} 1, a_{-} 2, \ldots\right.$

$\left.\hookrightarrow, a_{-} n\right)=\backslash \operatorname{mbox}\left\{\right.$ sequence of natural numbers with no prime ${ }^{\prime}$,

['theorem', 'primes ', 'sequence', 'no prime'], ['theorem', 'sequence' , 'no $\hookrightarrow$ prime'], [aab, aac])

aad.save()

$\operatorname{aad}()$

print (aad.tags)

print (aad.tags_name)

aad.d

saved

$\forall n \in \mathbb{N}_{>0}, \exists\left(a_{1}, a_{2}, \ldots, a_{n}\right)=$ sequence of natural numbers with no prime

['theorem', 'primes', 'sequence', 'no prime']

['theorem', 'sequence', 'no prime']

$[74]:\left[\_: a a b, \ldots: a a c\right]$

\subsection{AAE: Definição (símbolo)}

$\$ O$ símbolo := indica que o que aparece à esquerda é definido como o que aparece à direita.\$ 


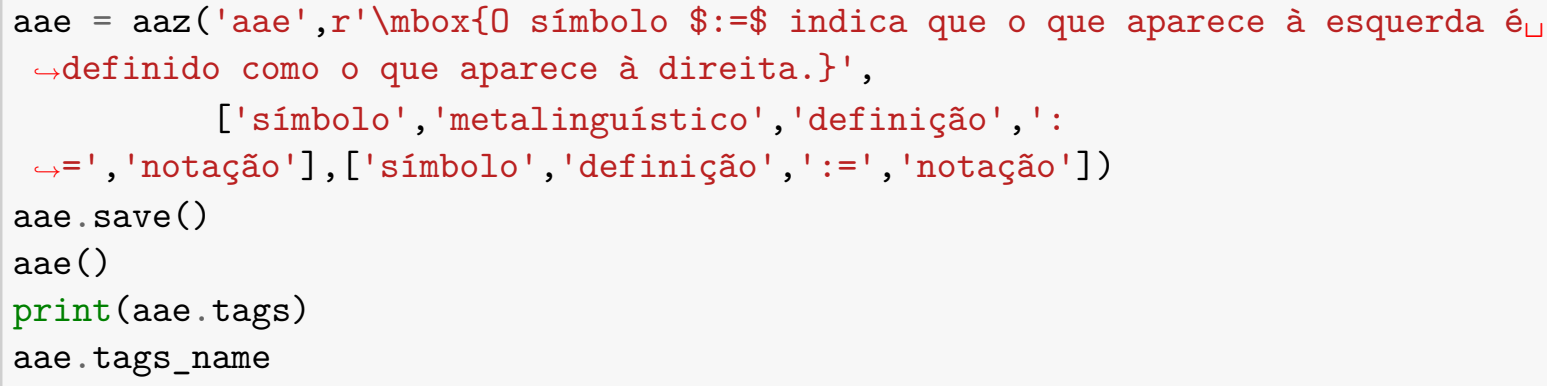

saved

O símbolo := indica que o que aparece à esquerda é definido como o que aparece à direita.

['símbolo', 'metalinguístico', 'definição', ':=', 'notação']

[18]: ['símbolo', 'definição', ':=', 'notação']

\subsection{AAF: Axioma}

axioma := verdade matemática evidente que não precisa ser demonstrada

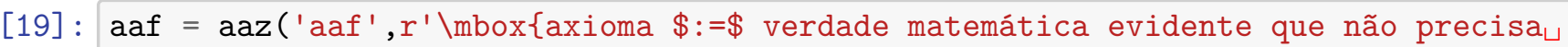
$\hookrightarrow$ ser demonstrada\}', ['axioma', 'definição'])

aaf . save()

$\operatorname{aaf}()$

aaf .tags

saved

axioma := verdade matemática evidente que não precisa ser demonstrada

[19]: ['axioma', 'definição']

\subsection{AAG: Sistema de Axiomas}

sistema de axiomas $:=$ conjunto de axiomas

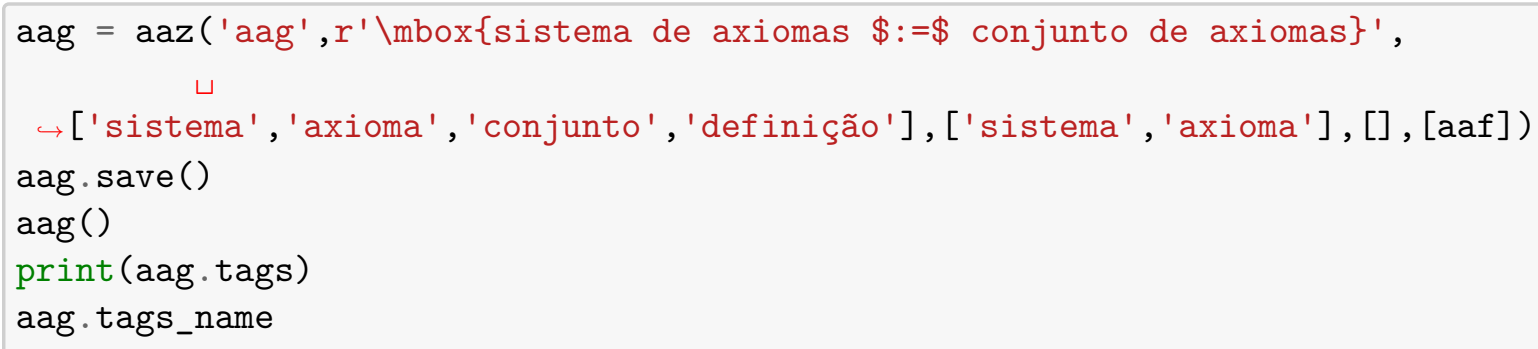

saved

sistema de axiomas $:=$ conjunto de axiomas

['sistema', 'axioma', 'conjunto', 'definição'] 
[20]: ['sistema', 'axioma']

\subsection{AAH: Estrutura matemática}

modelo := estrutura matemática

[21]: $a a h=\operatorname{aaz}\left({ }^{\prime} a a h ', r^{\prime} \backslash \operatorname{mbox}\{\right.$ modelo $\$:=\$$ estrutura matemática\}', ['modelo', 'estrutura' , 'estrutura $\hookrightarrow$ matemática', 'definição'], ['modelo'], [], [] )

aah.save()

$\operatorname{aah}()$

print (aah.tags)

aah.tags_name

saved

modelo $:=$ estrutura matemática

['modelo', 'estrutura', 'estrutura matemática', 'definição']

[21]: ['modelo']

\subsection{AAI: grupo (exemplo de modelo)}

Um grupo é um modelo (ou estrutura matemática) de um conjunto de axiomas.

[22]: aai = aaz('aai',r'\mbox\{Um grupo é um modelo (ou estrutura matemática) de um $\hookrightarrow$ conjunto de axiomas.\}',

['grupo' , 'modelo', 'estrutura' , 'estrutura $\sqcup$

$\hookrightarrow$ matemática' , 'exemplo'], ['grupo'], [] , [aah, aag, aaf])

aai.save()

aai()

print (aai.tags)

print (aai.tags_name)

aai.dep

saved

Um grupo é um modelo (ou estrutura matemática) de um conjunto de axiomas.

['grupo', 'modelo', 'estrutura', 'estrutura matemática', 'exemplo']

['grupo']

[22]: [_:aah, _:aag, _:aaf]

\subsection{AAJ: $\mathrm{R}^{\wedge} 3$ (exemplo de modelo)}

$\mathbb{R}^{3}$, que é uma coleção de trios ordenados de números reais,

é um modelo (ou estrutura matemática) de axiomas do espaço vetorial. 


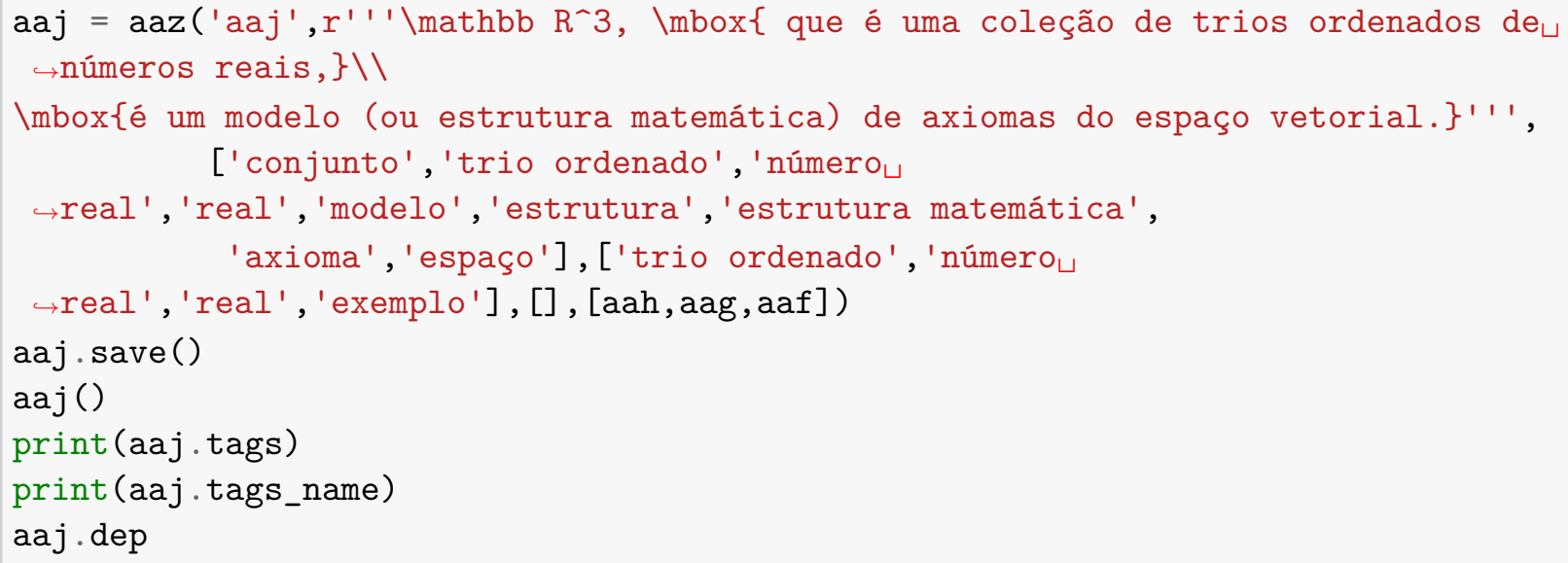

saved

$\mathbb{R}^{3}$, que é uma coleção de trios ordenados de números reais,

é um modelo (ou estrutura matemática) de axiomas do espaço vetorial.

['conjunto', 'trio ordenado', 'número real', 'real', 'modelo', 'estrutura', 'estrutura matemática', 'axioma', 'espaço']

['trio ordenado', 'número real', 'real', 'exemplo']

[84]: [_:aah, _:aag, _:aaf]

\subsection{AAK: Categorias de Símbolos}

\section{CATEGORIAS DE SÍMBOLO}

Parêntesis: $($,

Conectivos: $\vee, \neg$

Quantificador: $\forall$

Variáveis (uma para cada inteiro positivo $n$ ): $v_{1}, v_{2}, \ldots, v_{n}, \ldots$

Vars $:=$ conjunto de símbolos de variáveis

Igualdade: $=$

Constante: para cada $n$ inteiro positivo, algum conjunto contendo zero ou mais símbolos

Função: para cada $n$ inteiro positivo, algum conjunto contendo zero ou mais símbolos de funções $n$-árias

Relação: para cada $n$ inteiro positivo, algum conjunto contendo zero ou mais símbolos de relações $n$-árias

[90]: aak = aaz $\left({ }^{\prime} a k^{\prime}, r '\right.$ ' ' $\backslash \operatorname{mbox}\{C A T E G O R I A S$ DE SÍMBOLOS $\} \backslash \backslash$

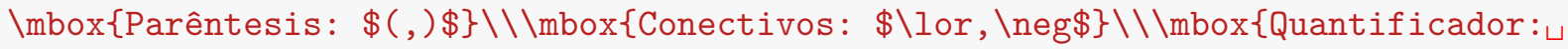
$\hookrightarrow \$ \backslash$ forall $\$\} \backslash \backslash$

$\backslash \operatorname{mbox}\left\{\right.$ Variáveis (uma para cada inteiro positivo $\$ \mathrm{n} \$$ ): $\$ \mathrm{v}_{-} 1, \mathrm{v}_{-} 2, \ldots, \mathrm{v} \_\mathrm{n}, \ldots$. $\left.\$\right\} \backslash \backslash$

$\backslash \operatorname{mbox}\{\operatorname{Vars} \$:=\$$ conjunto de símbolos de variáveis\} $\backslash \backslash \backslash \operatorname{mbox}\{$ Igualdade: $\$=\$\} \backslash \backslash$

$\backslash$ mbox\{Constante: para cada $\$ \mathrm{n} \$$ inteiro positivo, algum conjunto contendo zero $\hookrightarrow$ ou mais simbolos $\} \backslash \backslash$

$\backslash$ mbox\{Função: para cada $\$ n \$$ inteiro positivo, algum conjunto contendo zero ou $\hookrightarrow$ mais símbolos de funções \$n\$-árias\} $\backslash \backslash$ 


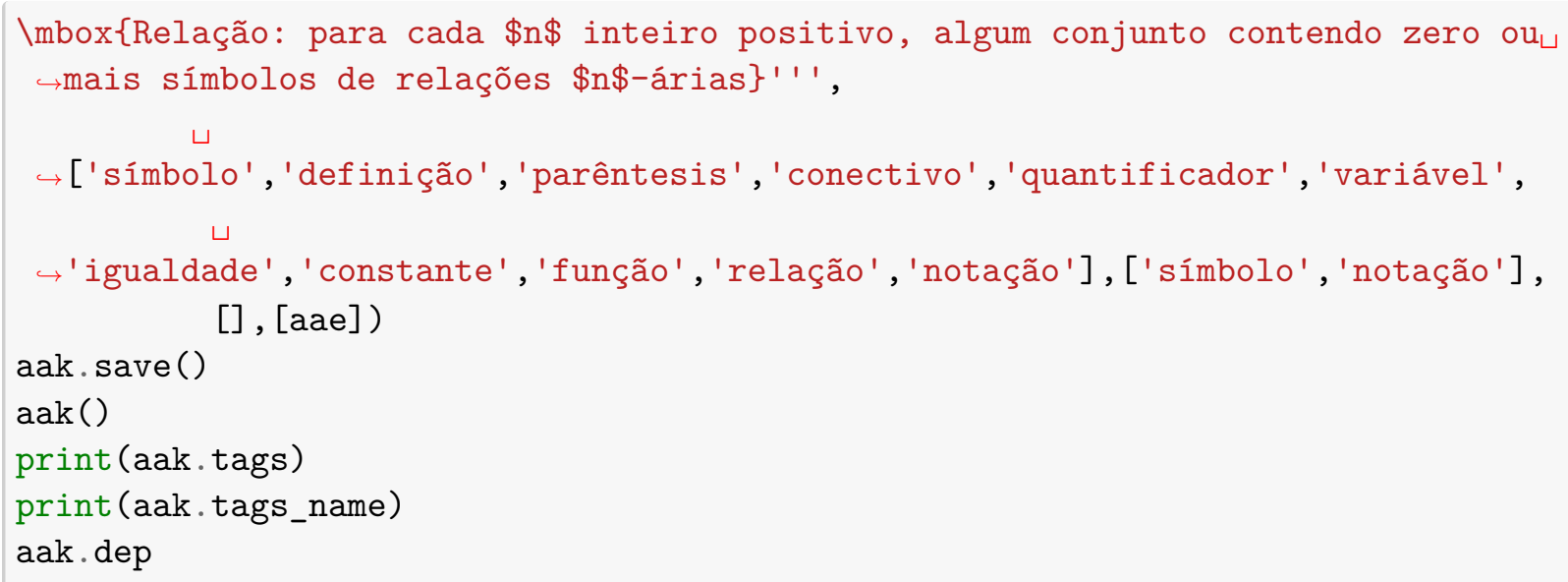

saved

\section{CATEGORIAS DE SÍMBOLOS}

Parêntesis: $($,

Conectivos: $\vee, \neg$

Quantificador: $\forall$

Variáveis (uma para cada inteiro positivo $n$ ): $v_{1}, v_{2}, \ldots, v_{n}, \ldots$

Vars := conjunto de símbolos de variáveis

Igualdade: $=$

Constante: para cada $n$ inteiro positivo, algum conjunto contendo zero ou mais símbolos

Função: para cada $n$ inteiro positivo, algum conjunto contendo zero ou mais símbolos de funções $n$-árias Relação: para cada $n$ inteiro positivo, algum conjunto contendo zero ou mais símbolos de relações $n$-árias

['símbolo', 'definição', 'parêntesis', 'conectivo', 'quantificador', 'variável',

'igualdade', 'constante', 'função', 'relação', 'notação']

['símbolo', 'notação']

[90]: [_:aae]

\subsection{AAL: Sintaxe de uma linguagem}

sintaxe $:=$ símbolos (de uma linguagem)

[25]: aal = aaz('aal', $r^{\prime} \backslash \operatorname{mbox}\{$ sintaxe $\$:=\$$ simbolos (de uma linguagem) $\}$ ',

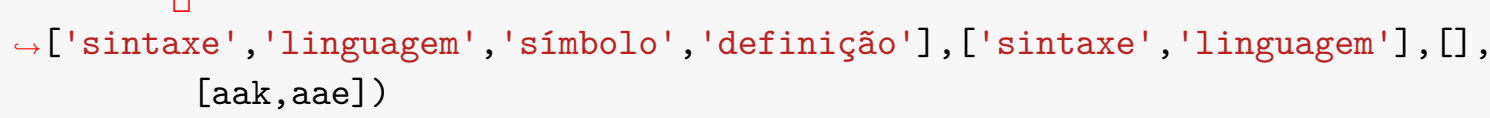

saved

sintaxe $:=$ símbolos (de uma linguagem) 
['sintaxe', 'linguagem', 'símbolo', 'definição']

['sintaxe', 'linguagem']

[25]: [_:aak, _:aae]

\subsection{AAM: Cadeia de Símbolos}

cadeia de símbolos $:=$ sequência de símbolos

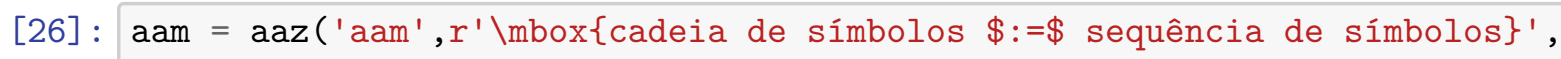
['cadeia', 'símbolo' , 'sequência' , 'definição'], ['cadeia' , 'símbolo'], [], [aak, aae] )

aam.save()

aam ()

print (aam.tags)

print (aam.tags_name)

aam.dep

saved

cadeia de símbolos $:=$ sequência de símbolos

['cadeia', 'símbolo', 'sequência', 'definição']

['cadeia', 'símbolo']

[26]: [_:aak, _:aae]

\subsection{AAN: Linguagem de Primeira Ordem}

linguagem de primeira ordem $:=$ coleção infinita de símbolos distintos (tal que nenhum esteja contido no outro)

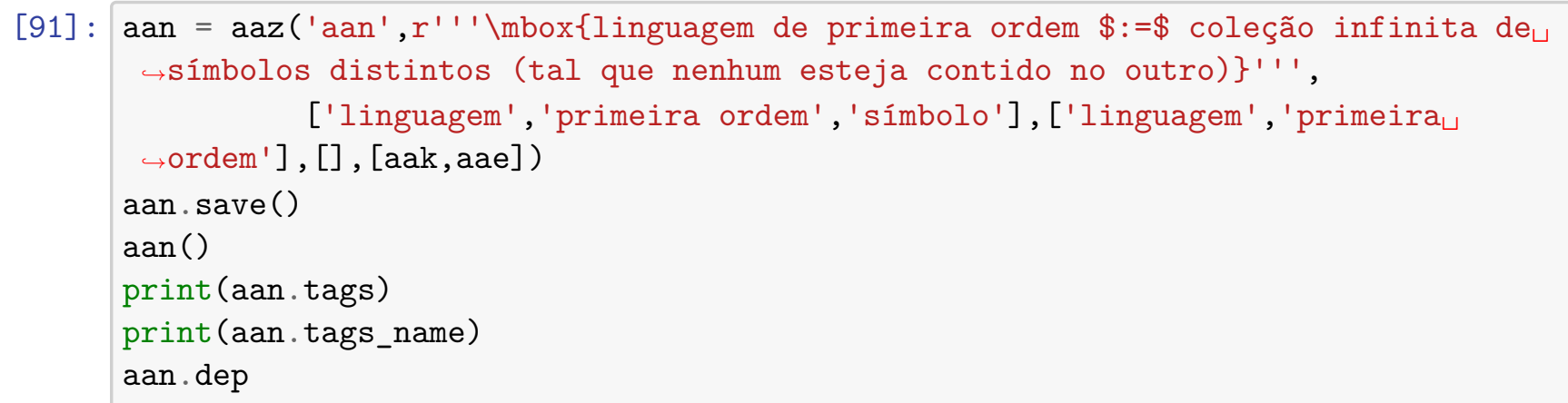

saved

linguagem de primeira ordem $:=$ coleção infinita de símbolos distintos (tal que nenhum esteja contido no outro)

['linguagem', 'primeira ordem', 'símbolo']

['linguagem', 'primeira ordem']

[91]: [_:aak, _:aae] 


\subsection{AAO: Função $n$-ária}

função $n$-ária := função de $n$ variáveis

[88]: aao = aaz('aao', r' \mbox\{função \$n\$-ária \$:=\$ função de \$n\$ variáveis\}', ['função' , 'n-ária ', 'aridade' , 'definição' ], ['função ' , 'n-ária' ], [] , [] )

aao.save()

aao ()

print (aao.tags)

print (aao.tags_name)

aao.dep

saved

função $n$-ária := função de $n$ variáveis

['função', 'n-ária', 'aridade', 'definição']

['função', 'n-ária']

[88] :

\subsection{AAP: Símbolo Mais (+)}

O símbolo + tem aridade 2, ou seja, é uma função de duas variáveis.

[29]: aap = aaz('aap', $r^{\prime} \backslash \operatorname{mbox}\{0$ símbolo $\$+\$$ tem aridade 2 , ou seja, é uma função de $\hookrightarrow$ duas variáveis.\}',

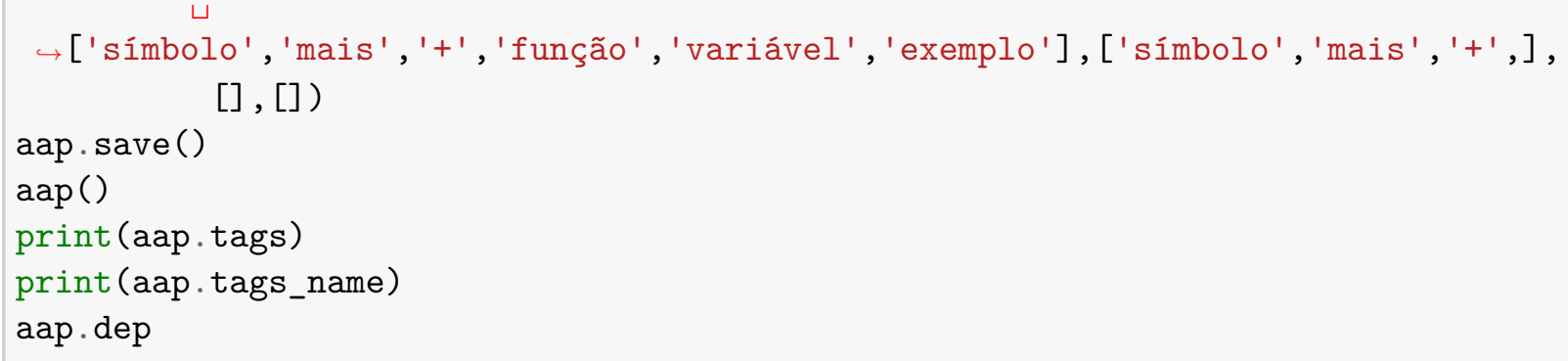

saved

O símbolo + tem aridade 2 , ou seja, é uma função de duas variáveis.

['símbolo', 'mais', '+', 'função', 'variável', 'exemplo']

['símbolo', 'mais', '+']

[29]:

\subsection{AAQ: $n$-upla}

$n$-upla $:=$ ?

[30]: aaq $=\operatorname{aaz}\left(\right.$ 'aaq $^{\prime}, r^{\prime} n \backslash \operatorname{mbox}\{-u p l a\}=? '$, ['n-upla', 'aridade', 'upla'], ['n-upla' , 'upla'], [] , [] )

aaq.save() 


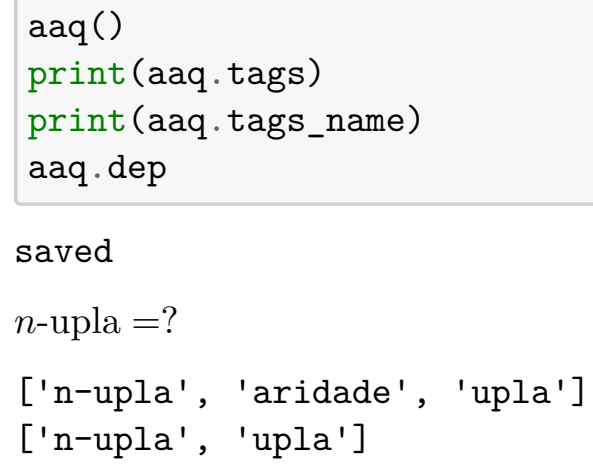

[30]:

\subsection{AAR: Relação $n$-ária}

relação $n$-ária $:=$ relação em $n$-uplas de objetos

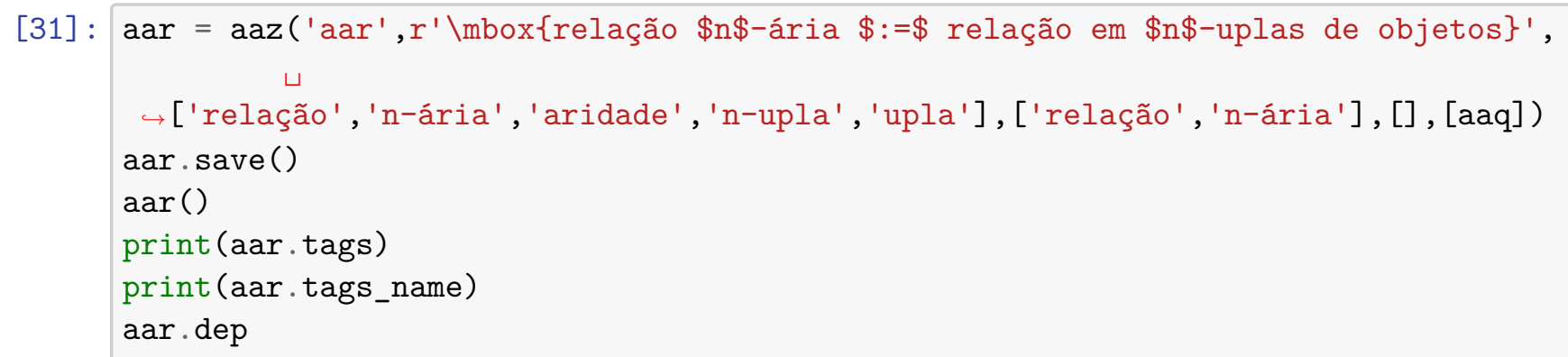

saved

relação $n$-ária $:=$ relação em $n$-uplas de objetos

['relação', 'n-ária', 'aridade', 'n-upla', 'upla']

['relação', 'n-ária']

[31]: [_:aaq]

\subsection{AAS: Especificar uma Linguagem}

especificar uma linguagem $:=$ listar os símbolos (constante, função, relação)

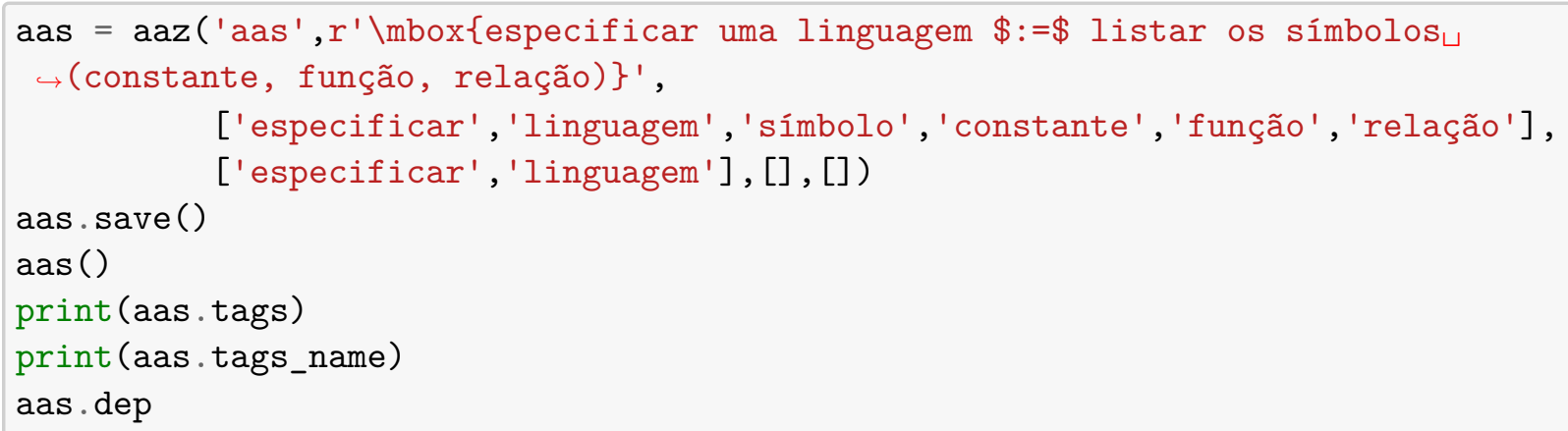


especificar uma linguagem := listar os símbolos (constante, função, relação)

['especificar', 'linguagem', 'símbolo', 'constante', 'função', 'relação']

['especificar', 'linguagem']

[32]: []

\subsection{AAT: Operação Binária}

operação binária $=$ ?

[33]: aat = aaz ('aat', r' \mbox\{operação binária $\$=\$$ ?\} ', ['operação binária'], ['operação binária'], [], [] )

aat.save()

aat ()

print (aat.tags)

print (aat.tags_name)

aat.dep

saved

operação binária $=$ ?

['operação binária']

['operação binária']

$[33]:[]$

\subsection{AAU: Identidade}

identidade $=$ ?

[34]: $\mathrm{aau}=\operatorname{aaz}\left({ }^{\prime} \mathrm{aau}{ }^{\prime}, \mathrm{r}^{\prime} \backslash \operatorname{mbox}\{\right.$ identidade $\$=\$$ ?\} ', ['identidade'], ['identidade'], [], [] )

aau.save()

aau ()

print (aau.tags)

print (aau.tags_name)

aau.dep

saved

identidade $=$ ?

['identidade']

['identidade']

[34]: []

\subsection{AAV: Inversa}

inversa $=$ ? 
[35]: $\operatorname{aav}=\operatorname{aaz}\left({ }^{\prime} \operatorname{aav}^{\prime}, r^{\prime} \backslash \operatorname{mbox}\{\right.$ inversa $\$=\$$ ? $\}$ ', ['inversa'], ['inversa'], [], [])

aav.save()

$\operatorname{aav}()$

print (aav.tags)

print (aav.tags_name)

aav.dep

saved

inversa $=$ ?

['inversa']

['inversa']

$[35]:[]$

\subsection{AAW: Grupo}

grupo $:=$ conjunto + operação binária + identidade + inversa

[36]: aaw $=\operatorname{aaz}\left(\right.$ 'aaw' $^{\prime} r^{\prime} \backslash \operatorname{mbox}\{$ grupo $\$:=\$$ conjunto $\$+\$$ operação binária $\$+\$$ identidade $\hookrightarrow+\$$ inversa\}',

['grupo', ' conjunto' , 'operaçãoப

$\hookrightarrow$ binária', 'identidade', 'inversa'], ['grupo'], [], [aat, aau, aav])

aaw. save()

aaw ()

print (aaw.tags)

print (aaw.tags_name)

aaw.dep

saved

grupo $:=$ conjunto + operação binária + identidade + inversa

['grupo', 'conjunto', 'operação binária', 'identidade', 'inversa']

['grupo']

[36]: [_:aat, _:aau, _:aav]

\subsection{AAX: Termos}

Termos são cadeias de símbolos que se referem aos objetos da linguagem.

[37]: $\operatorname{aax}=\operatorname{aaz}\left({ }^{\prime} a a x ', r^{\prime} \backslash \operatorname{mbox}\{\right.$ Termos são cadeias de símbolos que se referem aos $\sqcup$ $\hookrightarrow$ objetos da linguagem.\}',

['termo', 'cadeia' , 'símbolo' , 'linguagem '], ['termo'], [], [aan, aas])

aax.save()

$\operatorname{aax}()$

print (aax.tags)

print (aax.tags_name) 
aax $\cdot \operatorname{dep}$

saved

Termos são cadeias de símbolos que se referem aos objetos da linguagem.

['termo', 'cadeia', 'símbolo', 'linguagem']

['termo']

[37]: [_:aan, _:aas]

\subsection{AAY: $\mathcal{L}$-símbolos}

$\mathcal{L}$-símbolos significa os símbolos da linguagem $\mathcal{L}$.

[38]:

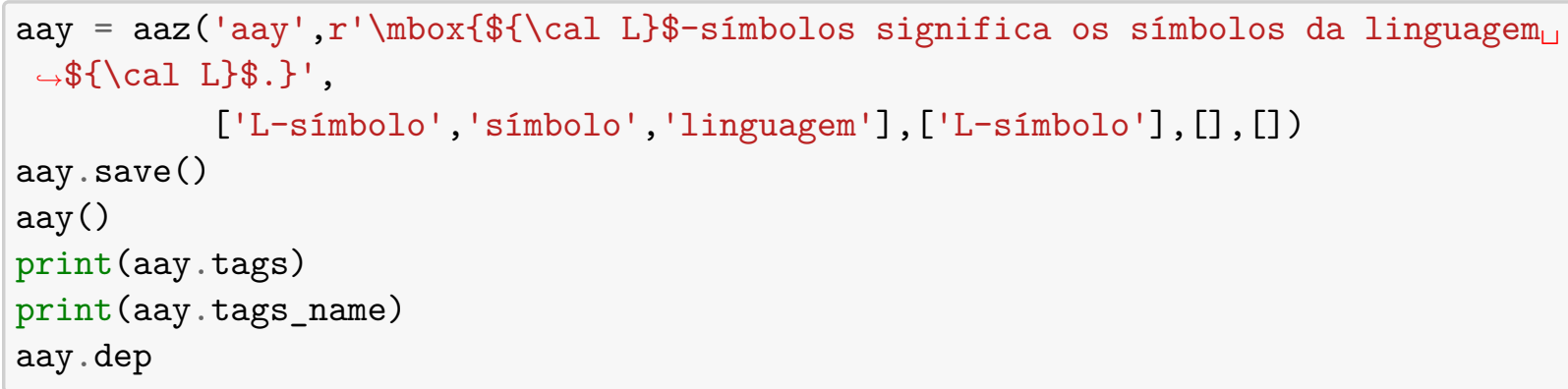

saved

$\mathcal{L}$-símbolos significa os símbolos da linguagem $\mathcal{L}$.

['L-símbolo', 'símbolo', 'linguagem']

['L-símbolo']

[38]:

\subsection{ABA: Símbolo :三}

O símbolo metalinguístico $: \equiv$ significa que as cadeias de $\mathcal{L}$-símbolos de cada lado de $: \equiv$ são idênticos

[75]: $a b a=a a z\left(' a b a ', r^{\prime} \backslash \operatorname{mbox}\{0\right.$ símbolo metalinguístico $\$: \backslash$ equiv\$ significa que as $\sqcup$ $\hookrightarrow$ cadeias de $\$\{\backslash$ cal L\}\$-símbolos de cada lado de $\$$ : \equiv\$ são idênticos.\}', ['símbolo' , 'metalinguístico' , ' : ', 'equivalente' , 'notação'], ['símbolo', 'metalinguístico', ' :

$\hookrightarrow$ ', 'equivalente' , 'notação' ], [], [aam, aay])

aba.save()

$\operatorname{aba}()$

print (aba.tags)

print (aba.tags_name)

aba.dep

saved

O símbolo metalinguístico : $\equiv$ significa que as cadeias de $\mathcal{L}$-símbolos de cada lado de $: \equiv$ são idênticos. 
['símbolo', 'metalinguístico', ': ', 'equivalente', 'notação']

['símbolo', 'metalinguístico', ': ', 'equivalente', 'notação']

$[75]: \quad\left[\_: a a m, \quad\right.$ _aay $]$

\subsection{ABB: Termo de uma Linguagem}

TERMO (de uma linguagem $\mathcal{L}$ ) $:=$ cadeia não vazia e finita de $t$ símbolos de $\mathcal{L}$ que satisfazem exatamente uma das seguintes condições:

(a) $t$ é uma variável, ou

(b) $t$ é um símbolo constante, ou

(c) $t: \equiv f t_{1} t_{2} \ldots t_{n}$, sendo $f$ um símbolo de função $n$-ária de $\mathcal{L}$ e cada um dos $t_{i}$ é um termo de $\mathcal{L}$.

[93] : $a b b=a a z\left(' a b b ', r^{\prime}{ }^{\prime} \backslash \operatorname{mbox}\{\right.$ TERMO (de uma linguagem $\$\{\backslash c a l ~ L\}):=\$$ cadeia nãoப $\hookrightarrow$ vazia e finita de $\$$ t\$ símbolos \}

$\backslash \operatorname{mbox}\{$ de $\$\{\backslash$ cal $L\} \$$ que satisfazem $\} \backslash \backslash \backslash \operatorname{mbox}\{$ exatamente uma das seguintes $\sqcup$ $\rightarrow$ condições: $\} \backslash \backslash$

$\backslash \operatorname{mbox}\{(\mathrm{a})$ \$t\$ é uma variável, ou $\} \backslash \backslash$

$\backslash \operatorname{mbox}\{(b) \$ t \$$ é um símbolo constante, ou $\backslash \backslash \backslash$

$\backslash \operatorname{mbox}\{(c)$ \$t: \equiv ft_1t_2...t_n\$, sendo $\$$ f $\$$ um símbolo de função $\$ n$ \$ária de

$\hookrightarrow \$\{\backslash$ cal L $\} \$$ e cada um dos $\$ t_{-} i \$$ é um termo de $\$\{\backslash c a l L\}$. $\}$ ''',

['termo', ' linguagem', 'cadeia', 'símbolo' , 'variável' , 'símbolo५

$\hookrightarrow$ constante' , ' constante' , 'função' , 'função n-ária' , 'n-ária' , 'aridade'],

['termo'] , [], [aax, aam, aan, aas, aak, aay, aba, aao])

abb.save()

$\mathrm{abb}()$

print (abb.tags)

print (abb.tags_name)

$a b b \cdot \operatorname{dep}$

saved

TERMO (de uma linguagem $\mathcal{L}$ ) := cadeia não vazia e finita de $t$ símbolos de $\mathcal{L}$ que satisfazem exatamente uma das seguintes condições:

(a) $t$ é uma variável, ou

(b) $t$ é um símbolo constante, ou

(c) $t: \equiv f t_{1} t_{2} \ldots t_{n}$, sendo $f$ um símbolo de função $n$-ária de $\mathcal{L}$ e cada um dos $t_{i}$ é um termo de $\mathcal{L}$.

['termo', 'linguagem', 'cadeia', 'símbolo', 'variável', 'símbolo constante', 'constante', 'função', 'função n-ária', 'n-ária', 'aridade']

['termo']

[93]: [_:aax, _:aam,_aan, _:aas, _:aak,_aay,_aba, _:aao]

\subsection{ABC: Fórmulas}

Fórmulas são afirmações acerca dos objetos de uma estrutura matemática (modelo). 


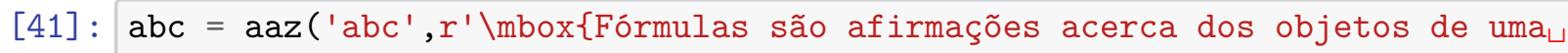
$\rightarrow$ estrutura matemática (modelo).\}',

['fórmula', 'modelo', 'estrutura

↔matemática', 'estrutura'], ['fórmula'], [], [aah])

abc.save()

$\operatorname{abc}()$

print (abc.tags)

print (abc.tags_name)

$a b c \cdot d e p$

saved

Fórmulas são afirmações acerca dos objetos de uma estrutura matemática (modelo).

['fórmula', 'modelo', 'estrutura matemática', 'estrutura']

['fórmula']

[41]: [_:aah]

\subsection{ABD: Notação Polonesa (exemplo)}

Exemplo de notação polonesa

$\cdot+\overline{3} \overline{2} \overline{4}: \equiv(\overline{3}+\overline{2}) \cdot \overline{4}: \equiv \overline{5} \cdot \overline{4}: \equiv \overline{20}$

[95]: $a b d=a a z\left(' a b d ', r^{\prime}\right.$ ''\mbox $\{$ Exemplo de notação polonesa\} $\backslash \backslash$

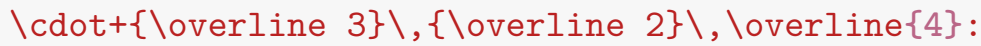

$\hookrightarrow$ lequiv (\overline $\{3\}+\backslash$ overline $\{2\}$ ) \cdot\overline $\{4\}$

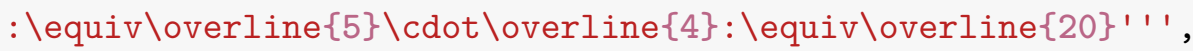

['notação polonesa', 'notação', 'exemplo', 'equivalente'],

['notação polonesa', 'notação', 'exemplo', 'equivalente '] , [] , [] )

abd.save()

$\operatorname{abd}()$

print (abd.tags)

print (abd.tags_name)

abd.dep

saved

Exemplo de notação polonesa

$\cdot+\overline{3} \overline{2} \overline{4}: \equiv(\overline{3}+\overline{2}) \cdot \overline{4}: \equiv \overline{5} \cdot \overline{4}: \equiv \overline{20}$

['notação polonesa', 'notação', 'exemplo', 'equivalente']

['notação polonesa', 'notação', 'exemplo', 'equivalente']

$[95]:[]$

\subsection{ABE: Função em notação polonesa}

Em notação polonesa, essas duas cadeias de símbolos são equivalentes, $f t_{1} t_{2} \ldots t_{n}: \equiv f\left(t_{1}, t_{2}, \ldots, t_{n}\right)$. 
[107]: abe = aaz('abe',r'''\mbox\{Em notação polonesa, essas duas cadeias de símbolos $\sqcup$ $\rightarrow$ são equivalentes, $\} \backslash \backslash$

ft_1t_2...t_n: \equiv $f\left(t_{-} 1, t_{-} 2, \ldots, t_{-} n\right) .1$ ' ,

['função' , ' notaçãoப

$\hookrightarrow$ polonesa' , 'notação' , 'cadeia' , 'símbolo' , 'equivalente'],

['função', 'notação polonesa', 'notação' , 'equivalente'], [], [])

abe.save()

abe ()

print (abe.tags)

print (abe.tags_name)

abe.dep

saved

Em notação polonesa, essas duas cadeias de símbolos são equivalentes,

$f t_{1} t_{2} \ldots t_{n}: \equiv f\left(t_{1}, t_{2}, \ldots, t_{n}\right)$.

['função', 'notação polonesa', 'notação', 'cadeia', 'símbolo', 'equivalente']

['função', 'notação polonesa', 'notação', 'equivalente']

[107]: []

\subsection{ABF: Fórmula (definição)}

FÓRMULA de uma linguagem $\mathcal{L}: \equiv$ cadeia $\phi$ não vazia e finita de símbolos de $\mathcal{L}$ que satisfazem exatamente uma das seguintes condições:

(a) $\phi: \equiv=t_{1} t_{2}$, sendo $t_{1}$ e $t_{2}$ termos de $\mathcal{L}$, ou

(b) $\phi: \equiv R t_{1} t_{2} \ldots t_{n}$, sendo $R$ um símbolo de uma relação $n$-ária de $\mathcal{L}$, e $t_{1}, t_{2}, \ldots, t_{n}$ são termos de $\mathcal{L}$, ou

(c) $\phi: \equiv(\neg \alpha)$, sendo $\alpha$ uma fórmula de $\mathcal{L}$, ou

(d) $\phi: \equiv(\alpha \vee \beta)$, sendo $\alpha$ e $\beta$ fórmulas de $\mathcal{L}$, ou

(e) $\phi: \equiv(\forall v)(\alpha)$, sendo $v$ uma variável e $\alpha$ uma fórmula de $\mathcal{L}$.

[102]: abf = aaz('abf',r'''\mbox\{FóRMULA de uma linguagem $\$\{\backslash c a l$ L $\}: \backslash$ equiv\$ cadeia

$\hookrightarrow \$ \backslash$ phi\$ não vazia e finita de símbolos de $\$\{\backslash c a l L\}\} \backslash \backslash$

$\backslash$ mbox\{que satisfazem exatamente uma das seguintes condições:\}\\

$\backslash \operatorname{mbox}\left\{(a) \$ \backslash p h i: \backslash e q u i v \sim=t \_1 t \_2 \$\right.$, sendo $\$ t_{-} 1 \$$ e $\$ t_{-} 2 \$$ termos de $\$\{\backslash c a l$ L $\}$, ou $\} \backslash \backslash$

$\backslash \operatorname{mbox}\{(b)$ \$phi: \equiv Rt_1t_2...t_n\$, sendo $\$ R \$$ um símbolo de uma relaçãoப

$\hookrightarrow \$ n \$$-ária de $\$\{\backslash$ cal L $\} \$$, e $\$ t_{-} 1 \$, \$ t_{-} 2 \$, \ldots, \$ t_{-} n \$$ são termos de $\$\{\backslash$ cal L $\}$, $\hookrightarrow$ ou $\} \backslash \backslash$

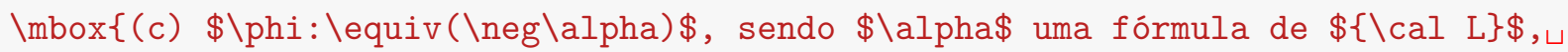
$\rightarrow$ ou $\} \backslash \backslash$

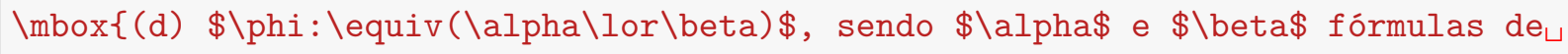
$\hookrightarrow \$\{\backslash c a l \mathrm{~L}\} \$$, ou $\} \backslash \backslash$

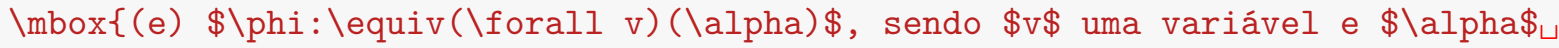
$\hookrightarrow$ uma fórmula de $\$\{\backslash c a l L\}$ L.\}\\}

$\backslash \operatorname{mbox}\{($ dizemos que o alcance do quantificador $\$ \backslash$ forall\$ é $\$ \backslash$ alpha\$)\}' ' ,

$\hookrightarrow[$ 'fórmula' , 'definição', ' linguagem ', 'cadeia' , ' símbolo' , ' termo ', 'relação' , 


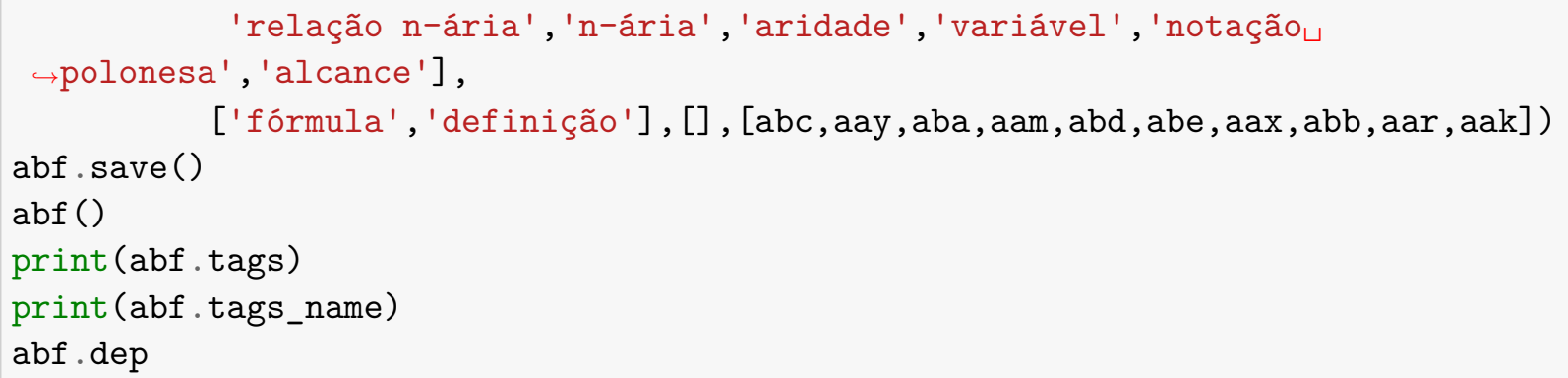

saved

FÓRMULA de uma linguagem $\mathcal{L}: \equiv$ cadeia $\phi$ não vazia e finita de símbolos de $\mathcal{L}$ que satisfazem exatamente uma das seguintes condições:

(a) $\phi: \equiv=t_{1} t_{2}$, sendo $t_{1}$ e $t_{2}$ termos de $\mathcal{L}$, ou

(b) $\phi: \equiv R t_{1} t_{2} \ldots t_{n}$, sendo $R$ um símbolo de uma relação $n$-ária de $\mathcal{L}$, e $t_{1}, t_{2}, \ldots, t_{n}$ são termos de $\mathcal{L}$, ou

(c) $\phi: \equiv(\neg \alpha)$, sendo $\alpha$ uma fórmula de $\mathcal{L}$, ou

(d) $\phi: \equiv(\alpha \vee \beta)$, sendo $\alpha$ e $\beta$ fórmulas de $\mathcal{L}$, ou

(e) $\phi: \equiv(\forall v)(\alpha)$, sendo $v$ uma variável e $\alpha$ uma fórmula de $\mathcal{L}$.

(dizemos que o alcance do quantificador $\forall$ é $\alpha$ )

['fórmula', 'definição', 'linguagem', 'cadeia', 'símbolo', 'termo', 'relação', 'relação n-ária', 'n-ária', 'aridade', 'variável', 'notação polonesa', 'alcance']

['fórmula', 'definição']

[102]: [_:abc, _:aay, _:aba, _:aam, _:abd, _:abe, _:aax, _:abb, _:aar, _:aak]

\subsection{ABG: Recursão}

recursão := fórmula que contém outra(s) fórmula(s)

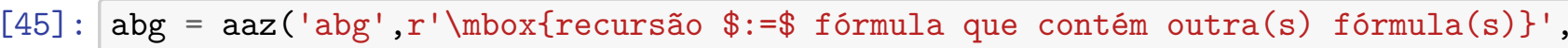
['recursão' , 'fórmula'] , ['recursão'], [] , [] )

abg.save()

$\operatorname{abg}()$

print (abg.tags)

print (abg.tags_name)

abg. dep

saved

recursão := fórmula que contém outra(s) fórmula(s)

['recursão', 'fórmula']

['recursão']

[45]:

\subsection{ABH: Fórmula Atômica}

fórmula atômica (mais simples) := primitiva (não recursiva) e tem 0 conectivo/quantificador 
[108]: $a b h=a a z\left(' a b h ', r^{\prime} \backslash \operatorname{mbox}\{\right.$ fórmula atômica (mais simples) $\$:=\$$ primitiva (nãoப $\hookrightarrow$ recursiva) e tem 0 conectivo/quantificador\}', ['fórmula

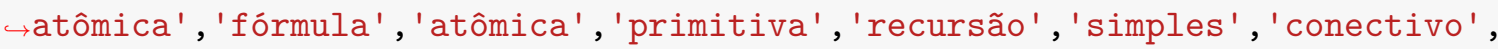
'quantificador'], ['fórmula atômica' , 'fórmula', 'atômica'], [], [abl])

abh.save()

$\operatorname{abh}()$

print (abh.tags)

print (abh.tags_name)

abh.dep

saved

fórmula atômica (mais simples) $:=$ primitiva (não recursiva) e tem 0 conectivo/quantificador

['fórmula atômica', 'fórmula', 'atômica', 'primitiva', 'recursão', 'simples', 'conectivo', 'quantificador']

['fórmula atômica', 'fórmula', 'atômica']

[108]: [

\subsection{ABI: Caso Base $(\mathrm{n}=1)$}

caso base: $n=1$

[47]: $a b i=a a z\left(' a b i ', r^{\prime} \backslash \operatorname{mbox}\{\right.$ caso base: $\$ \mathrm{n}=1 \$\}$ ', ['caso base', 'prova', 'indução'], ['caso base ', 'indução '], [], [] )

abi.save()

abi()

print (abi.tags)

print (abi.tags_name)

abi.dep

saved

caso base: $n=1$

['caso base', 'prova', 'indução']

['caso base', 'indução']

$[47]:[]$

\subsection{ABJ: Caso Indutivo $(\mathrm{k}>=1)$}

caso indutivo: $k \geq 1$

O raciocínio envolvido no caso indutivo é o da recursão.

[48]: $\operatorname{abj}=\operatorname{aaz}\left({ }^{\prime} a b j ', r^{\prime} \backslash \operatorname{mbox}\{c a s o\right.$ indutivo: \$k\geq1\$\}',

['caso indutivo', 'prova', 'indução'], ['caso৬

↔indutivo' , 'indução' ] , [] , [abg] )

abj.save() 


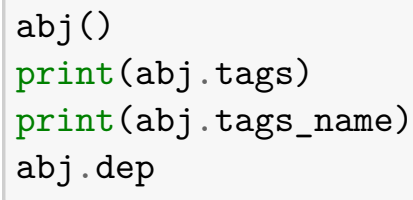

saved

caso indutivo: $k \geq 1$

['caso indutivo', 'prova', 'indução']

['caso indutivo', 'indução']

\section{$[48]: \quad[a: a b g]$}

\subsection{ABK: Indução na Complexidade da Fórmula/Termo}

INDUÇÃO na COMPLEXIDADE da FÓRMULA/TERMO

caso base $=$ fórmulas atômicas

caso indutivo $=$ fórmulas recursivas

[103] $:$ abk $=$ aaz $(' a b k ', r ' \prime \prime \backslash \operatorname{mbox}\{$ INDUÇÃO na COMPLEXIDADE da FÓRMULA/TERMO $\backslash \backslash$

$\backslash \operatorname{mbox}\{$ caso base $\$=\$$ fórmulas atômicas $\} \backslash \backslash \backslash \operatorname{mbox}\{$ caso indutivo $\$=\$$ fórmulas $\sqcup$

$\hookrightarrow$ recursivas\}' ' ',

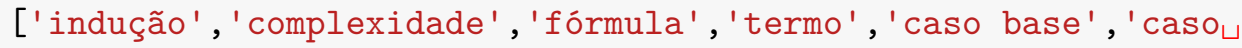
$\hookrightarrow$ indutivo'],

['indução' , ' complexidade' , 'fórmula' , 'termo'],

[aab, abi , abj , abc, abf , abh] , [aab, abi , abj, abc, abf , abh])

abk.save()

abk()

print (abk.tags)

print (abk.tags_name)

abk.dep

saved

INDUÇÃO na COMPLEXIDADE da FÓRMULA/TERMO

caso base $=$ fórmulas atômicas

caso indutivo $=$ fórmulas recursivas

['indução', 'complexidade', 'fórmula', 'termo', 'caso base', 'caso indutivo']

['indução', 'complexidade', 'fórmula', 'termo']

[103]: [_:aab, _:abi, _:abj, _:abc, _:abf, _:abh]

\subsection{ABL: Fórmula mais simples}

fórmula mais simples := número menor de conectivos/quantificadores

Se $\alpha$ é uma subfórmula da fórmula $\phi$, então $\alpha$ é mais simples do que $\phi$. 


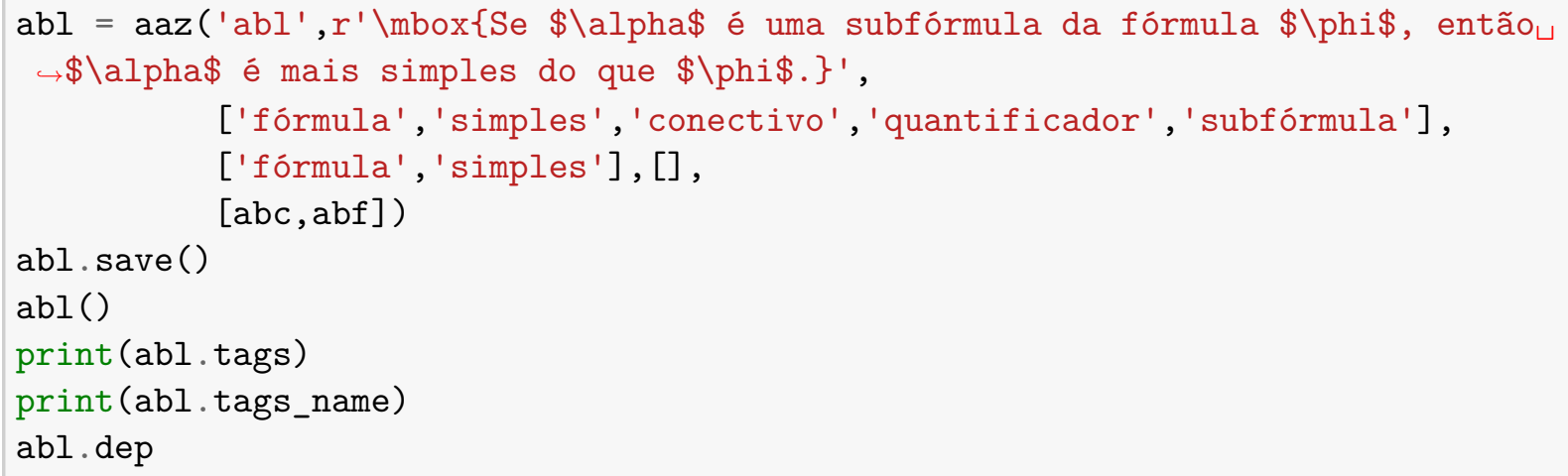

saved

Se $\alpha$ é uma subfórmula da fórmula $\phi$, então $\alpha$ é mais simples do que $\phi$.

['fórmula', 'simples', 'conectivo', 'quantificador', 'subfórmula']

['fórmula', 'simples']

[104]: [_:abc, _:abf]

\subsection{ABM: Indução na Estrutura da Fórmula}

\section{INDUÇÃO na ESTRUTURA da FÓRMULA/TERMO}

caso base $=$ fórmula mais simples (número menor de conectivos/quantificadores) caso indutivo $=$ indução nos números naturais

[105]: $a b k=a a z(' a b k ', r '$ ' '\mbox\{INDUÇÃO na ESTRUTURA da FÓRMULA/TERMO $\backslash \backslash$

$\backslash \operatorname{mbox}\{$ caso base $\$=\$$ fórmulas atômicas $\} \backslash \backslash \backslash \operatorname{mbox}\{$ caso indutivo $\$=\$$ fórmulas $\sqcup$

$\hookrightarrow$ recursivas\}' ' ',

['indução', 'estrutura', 'fórmula' , 'caso base ', 'caso

$\hookrightarrow$ indutivo', 'conectivo', 'quantificador'],

['indução' , 'estrutura' , 'fórmula'],

[aab, abi , abj , abc, abf , abh] , [aab, abi , abj , abc, abf , abh])

abk.save()

abk ()

print (abk.tags)

print (abk.tags_name)

abk.dep

saved

INDUÇÃO na ESTRUTURA da FÓRMULA/TERMO

caso base $=$ fórmulas atômicas

caso indutivo $=$ fórmulas recursivas

['indução', 'estrutura', 'fórmula', 'caso base', 'caso indutivo', 'conectivo', 'quantificador']

['indução', 'estrutura', 'fórmula']

[105]: [_:aab, _:abi, _:abj, _:abc, _:abf, _:abh] 


\subsection{ABN: Complexidade de uma Fórmula}

complexidade de uma fórmula := número de subfórmulas

[65]: $a b n=a a z\left(' a b n ', r^{\prime} \backslash \operatorname{mbox}\{\right.$ complexidade de uma fórmula $\$:=\$$ número de $\sqcup$ $\hookrightarrow$ subfórmulas\}' ',

['complexidade' , 'fórmula' , 'subfórmula '], ['complexidade' , 'fórmula '] , [] , $[a b c, a b f])$

abn.save()

abn ()

print (abn.tags)

print (abn.tags_name)

abn.dep

saved

complexidade de uma fórmula $:=$ número de subfórmulas

['complexidade', 'fórmula', 'subfórmula']

['complexidade', 'fórmula']

$[65]: \quad\left[\_: a b c, \ldots: a b f\right]$

\subsection{ABO: Estrutura de uma Fórmula}

estrutura de uma fórmula $:=$ número de conectivos/quantificadores

[66]: abo = aaz('abo',r'\mbox\{estrutura de uma fórmula \$:=\$ número de conectivos/

$\hookrightarrow$ quantificadores\}',

$\hookrightarrow$ ['estrutura' , 'fórmula', 'conectivo', 'quantificador'], ['estrutura' , 'fórmula'], [], $[\mathrm{abc}, \mathrm{abf}])$

abo.save()

abo()

print (abo.tags)

print (abo.tags_name)

abo.dep

saved

estrutura de uma fórmula $:=$ número de conectivos/quantificadores

['estrutura', 'fórmula', 'conectivo', 'quantificador']

['estrutura', 'fórmula']

$[66]:\left[\_: a b c, \ldots: a b f\right]$

$[$ ] : 


\section{omkb}

January 17, 2021

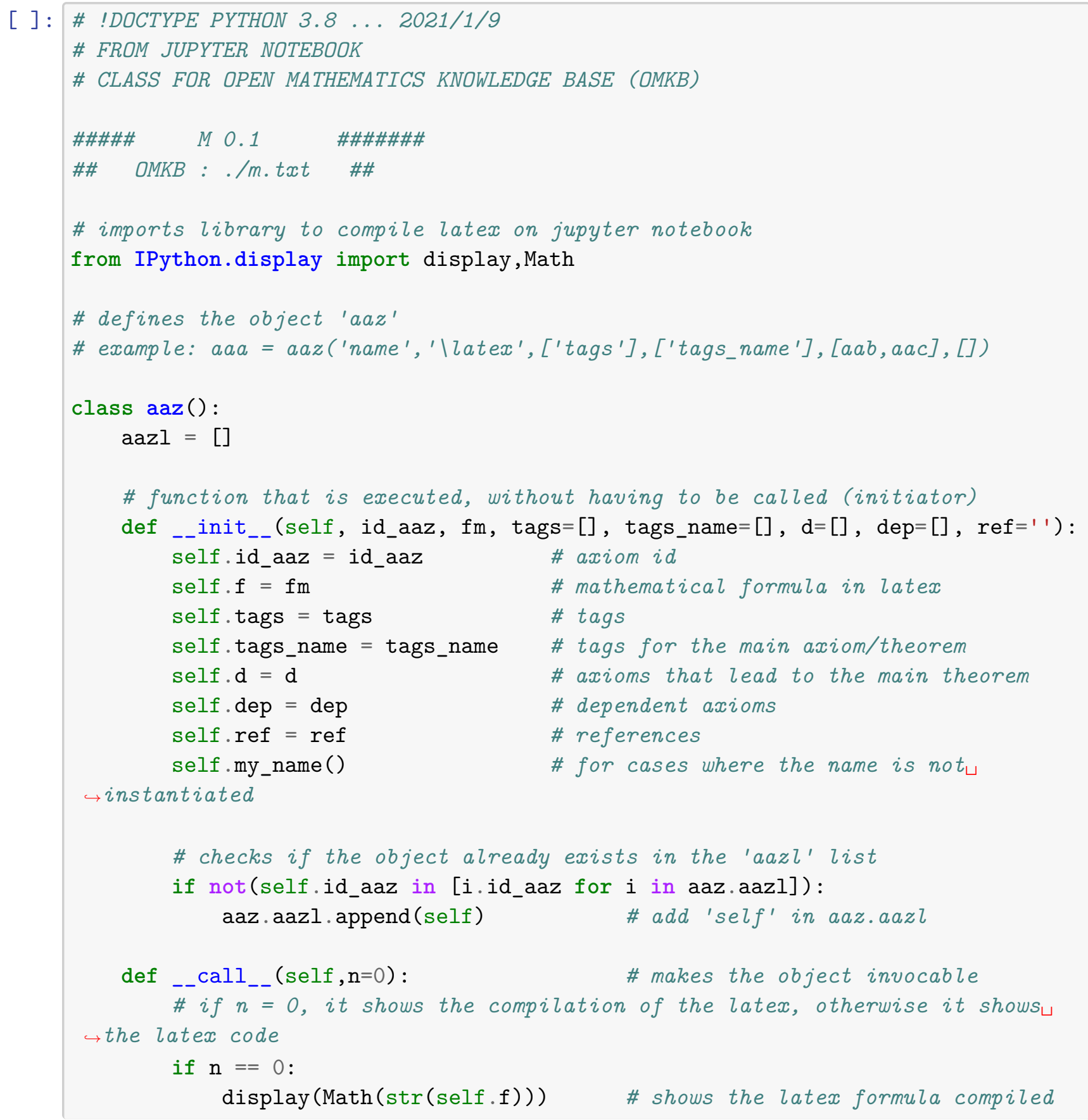




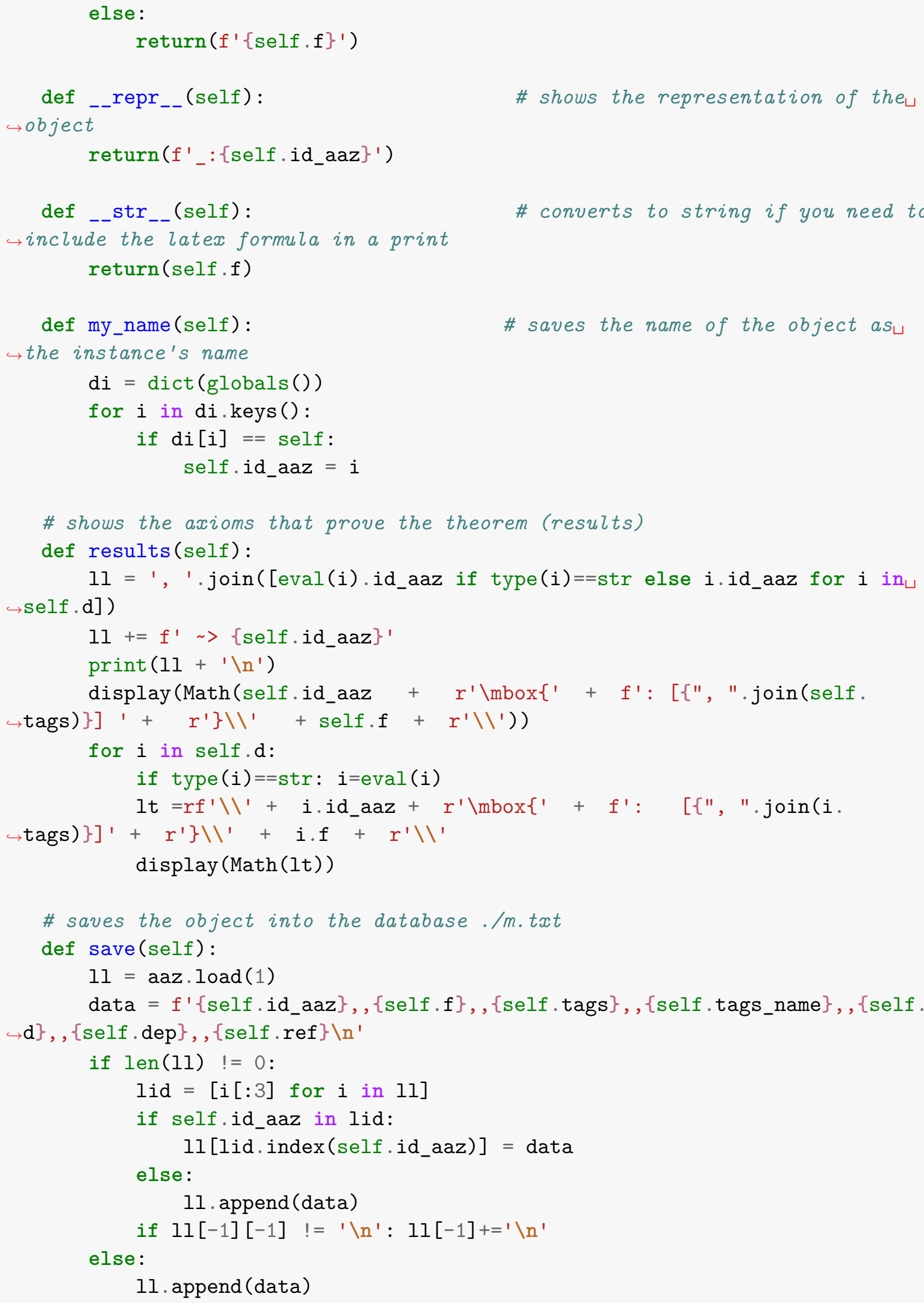




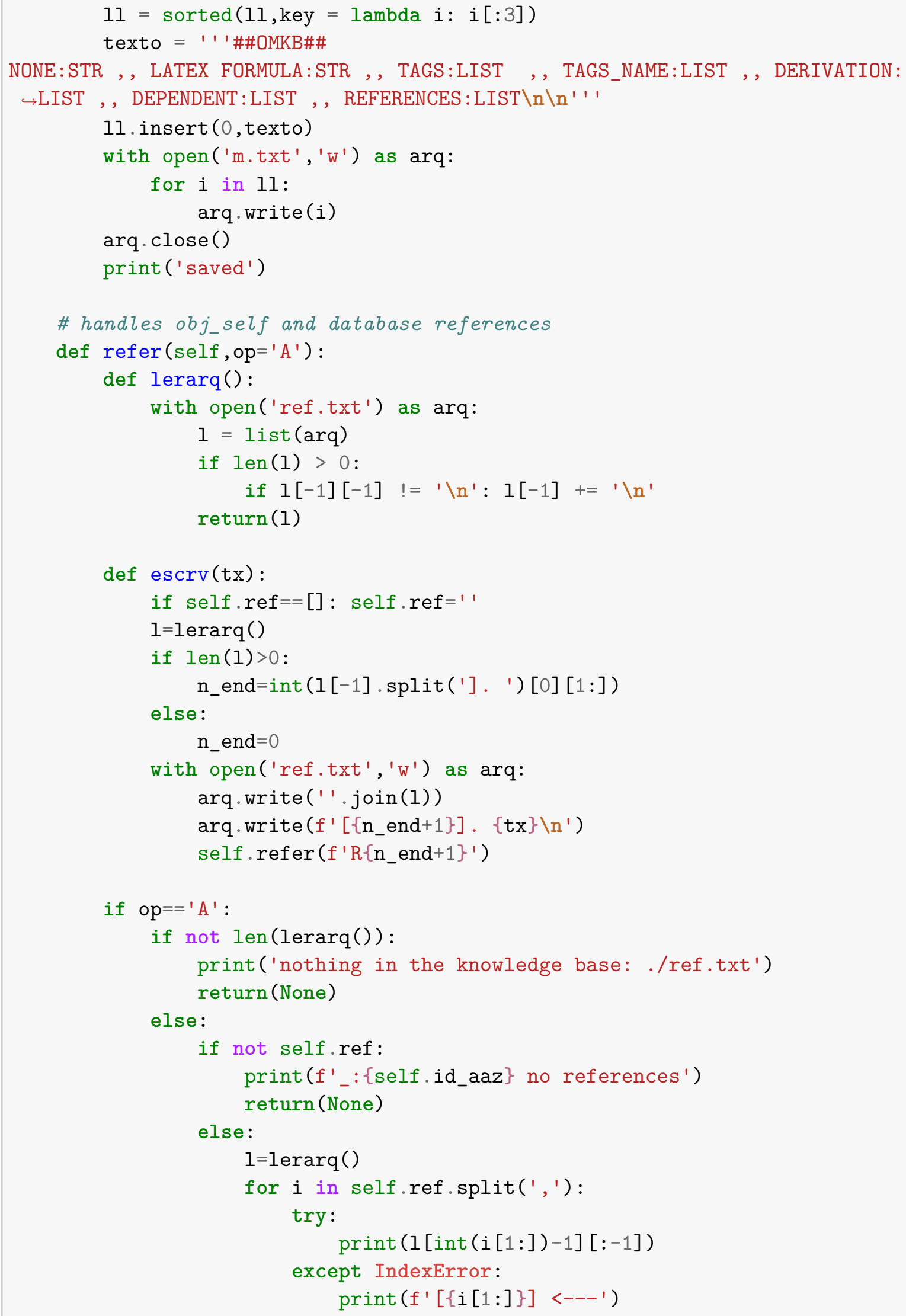




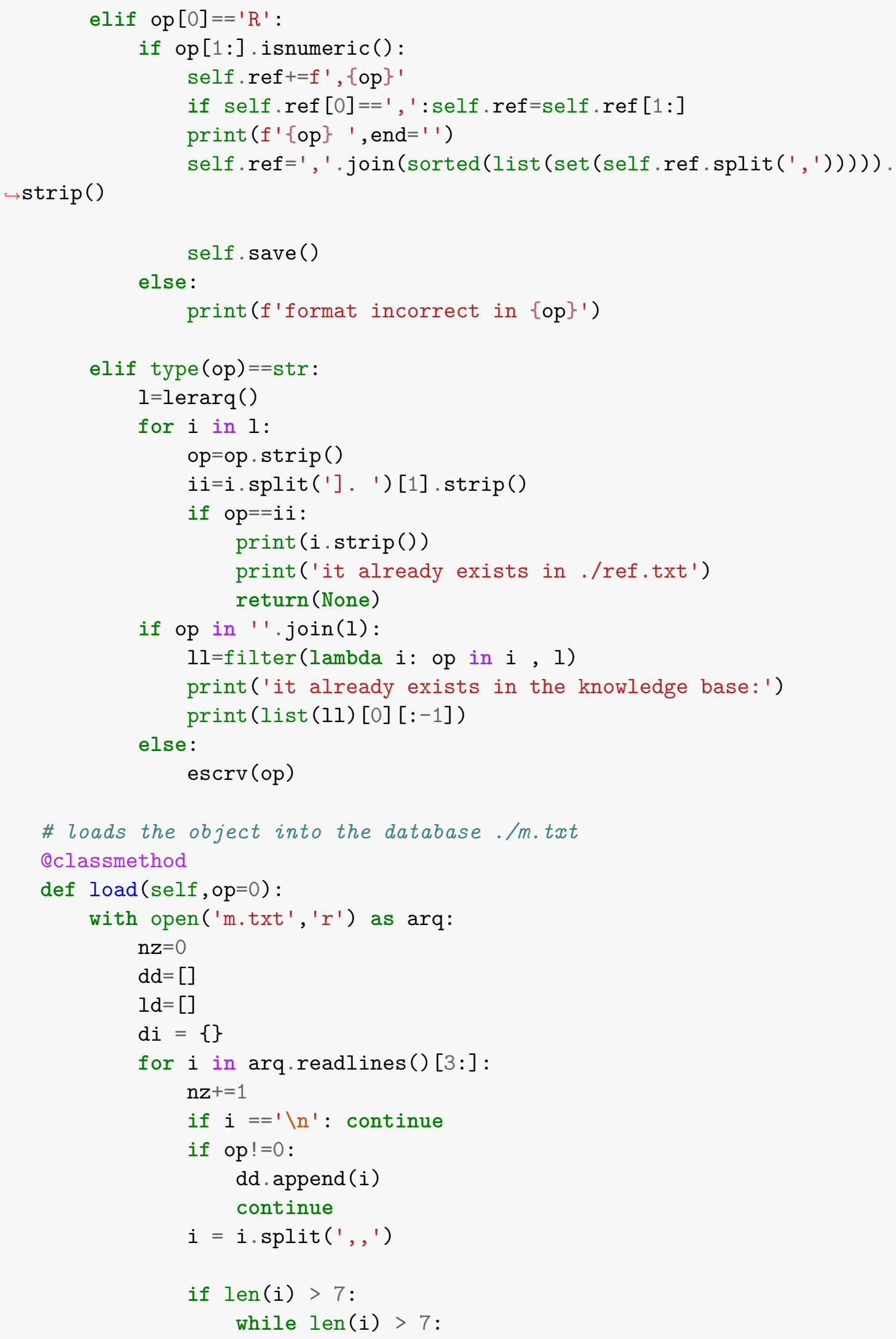




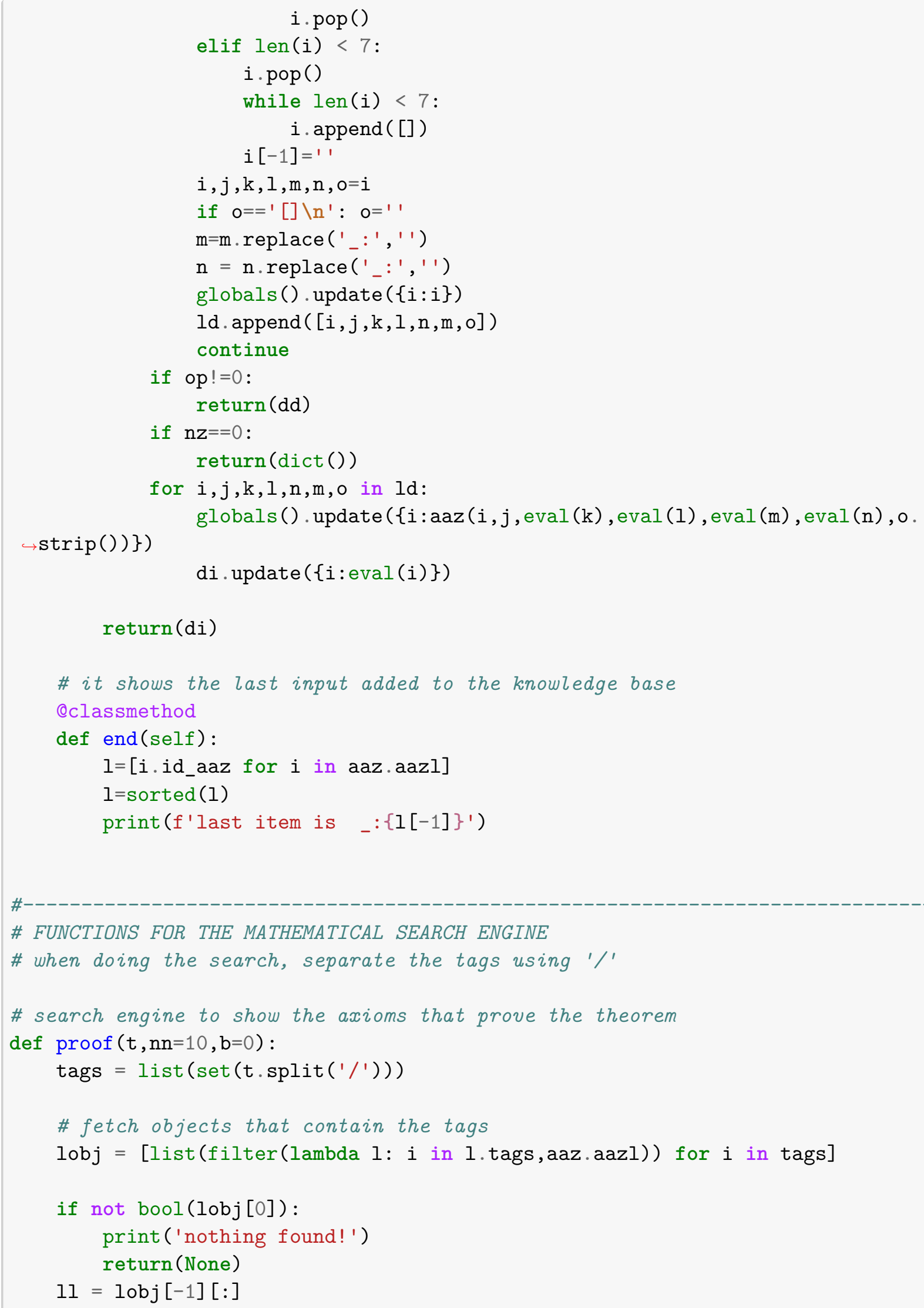




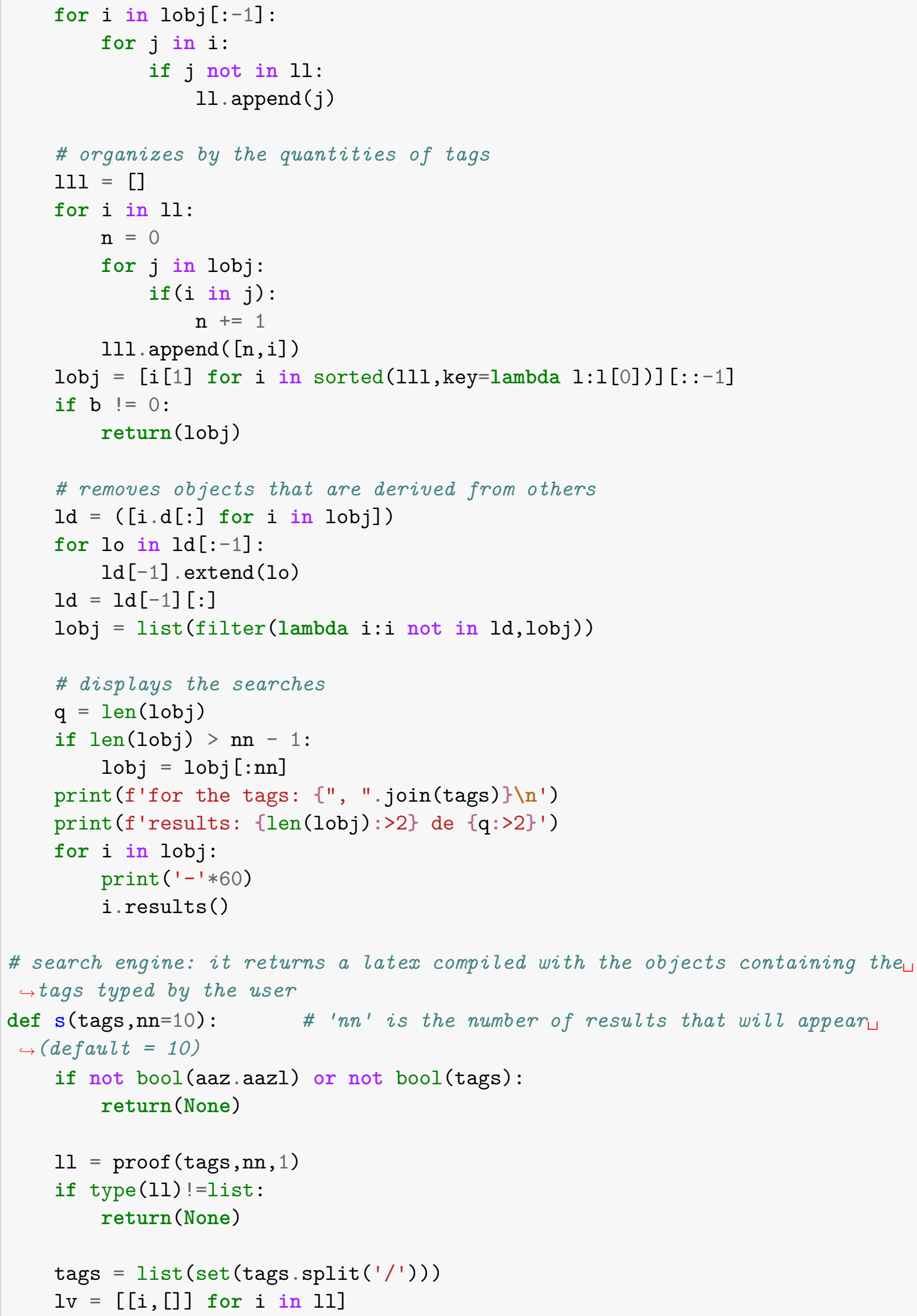




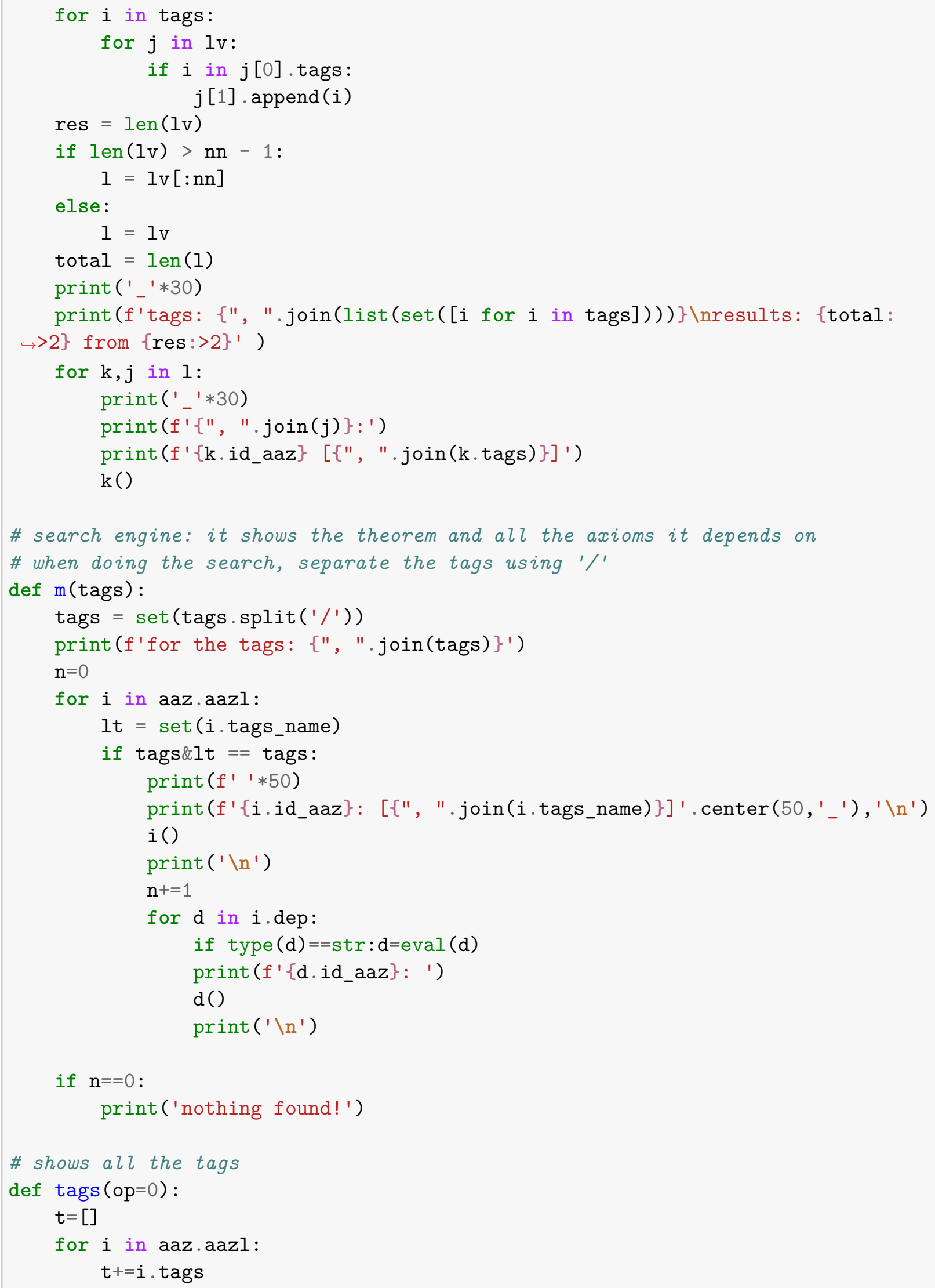




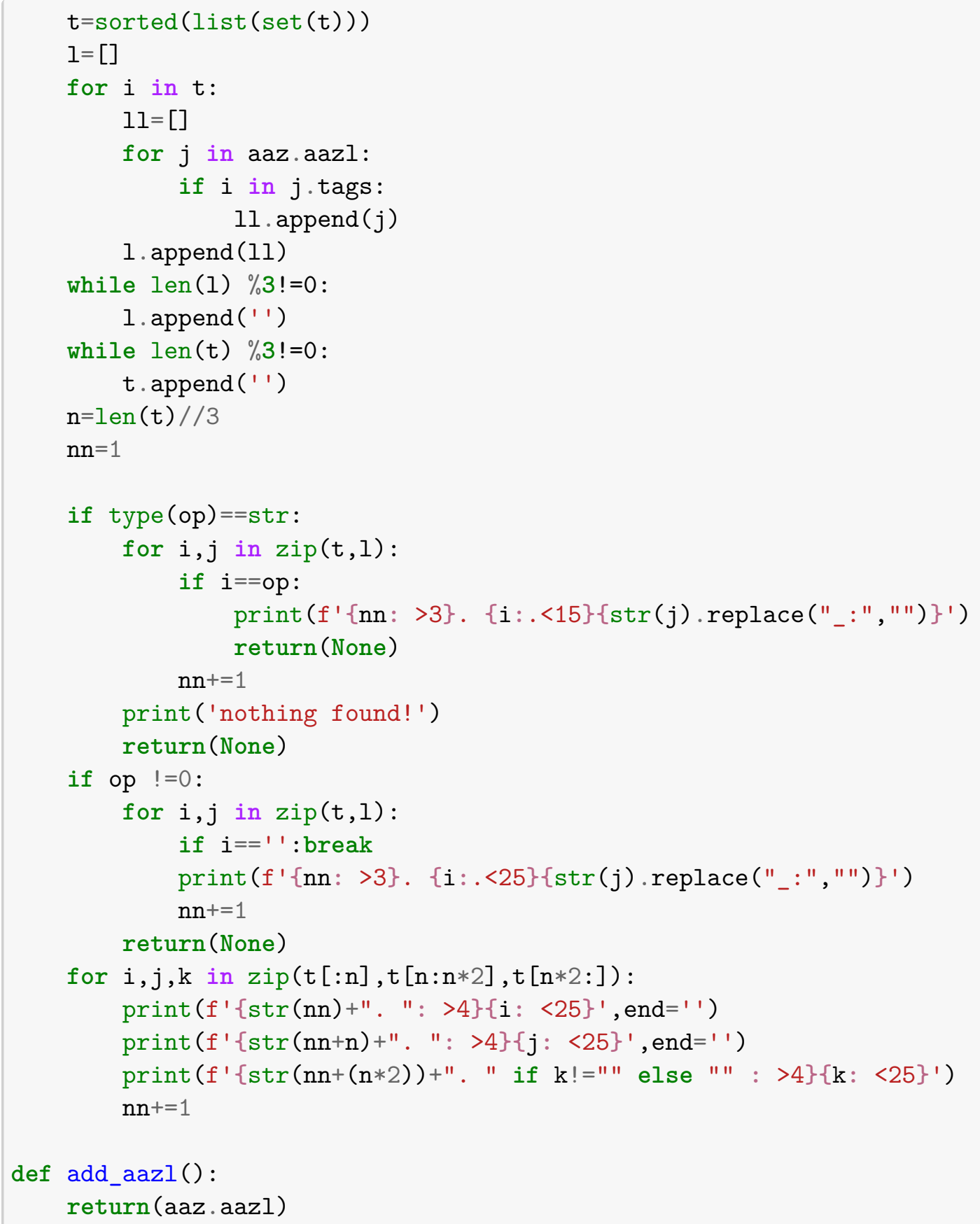

